\title{
Dopaminergic Contributions to Vocal Learning
}

\author{
Lukas A. Hoffmann, ${ }^{1,2}$ Varun Saravanan, ${ }^{1,2}{ }^{-A}$ Alynda N. Wood, ${ }^{1,2}{ }^{\circ L i} \mathrm{He},{ }^{3}$ and Samuel J. Sober ${ }^{2}$ \\ ${ }^{1}$ Neuroscience Doctoral Program, ${ }^{2}$ Department of Biology, and ${ }^{3}$ Department of Ophthalmology, Emory University, Atlanta, Georgia 30322
}

\begin{abstract}
Although the brain relies on auditory information to calibrate vocal behavior, the neural substrates of vocal learning remain unclear. Here we demonstrate that lesions of the dopaminergic inputs to a basal ganglia nucleus in a songbird species (Bengalese finches, Lonchura striata var. domestica) greatly reduced the magnitude of vocal learning driven by disruptive auditory feedback in a negative reinforcement task. These lesions produced no measureable effects on the quality of vocal performance or the amount of song produced. Our results suggest that dopaminergic inputs to the basal ganglia selectively mediate reinforcement-driven vocal plasticity. In contrast, dopaminergic lesions produced no measurable effects on the birds' ability to restore song acoustics to baseline following the cessation of reinforcement training, suggesting that different forms of vocal plasticity may use different neural mechanisms.
\end{abstract}

Key words: basal ganglia; Bengalese finch; dopamine; negative reinforcement; songbird; vocal learning

\section{Significance Statement}

During skill learning, the brain relies on sensory feedback to improve motor performance. However, the neural basis of sensorimotor learning is poorly understood. Here, we investigate the role of the neurotransmitter dopamine in regulating vocal learning in the Bengalese finch, a songbird with an extremely precise singing behavior that can nevertheless be reshaped dramatically by auditory feedback. Our findings show that reduction of dopamine inputs to a region of the songbird basal ganglia greatly impairs vocal learning but has no detectable effect on vocal performance. These results suggest a specific role for dopamine in regulating vocal plasticity.

\section{Introduction}

The brain relies on sensory information to guide the acquisition and maintenance of complex motor skills, including vocal behavior. Neurophysiological studies in mammals have shown that the activity of midbrain dopamine (DA) neurons reflects the difference between predicted and experienced reward, leading to the widespread hypothesis that such signals guide reinforcement learning (Schultz, 2007, 2013). However, establishing DA's role in sensorimotor learning, including vocal learning, has proved challenging in both experimental and clinical settings. Neurotoxic lesions of DA inputs to the basal ganglia often result in severe motor performance deficits (Zhou and Palmiter, 1995;

\footnotetext{
Received Oct. 27, 2015; revised Dec. 14, 2015; accepted Jan. 5, 2016.

Author contributions: L.A.H. and S.J.S. designed research; L.A.H., V.S., A.N.W., and L.H. performed research; L.A.H., V.S., A.N.W., L.H., and S.J.S. analyzed data; L.A.H., V.S., A.N.W., and S.J.S. wrote the paper.

This work was supported by National Institutes of Health National Institute of Neurological Disorders and Stroke R01 NS084844, National Institutes of Health National Institute of Neurological Disorders and Stroke F31NS089406, National Institutes of Health Computational Neuroscience Training Grant 5R90DA033462, Emory's Institute for Quantitative Theory and Methods, Emory's Udall Center of Excellence for Parkinson's Disease Research, and Emory's Integrated Cellular Imaging Microscopy Core. We thank Xiang Cheng (Emory University, Physics Department) for statistical assistance.

The authors declare no competing financial interests.

Correspondence should be addressed to Dr. Samuel Sober, 1510 Clifton Road NE, Room 2006, Atlanta, GA 30322. E-mail: samuel.j.sober@emory.edu.

DOI:10.1523/JNEUROSCI.3883-15.2016

Copyright $\odot 2016$ the authors $\quad 0270-6474 / 16 / 362176-14 \$ 15.00 / 0$
}

Beeler et al., 2010), complicating efforts to isolate DA's specific contributions to motor learning. Moreover, because the mammalian striatum mediates a wide range of behaviors, dopaminergic inputs likely affect cognitive and behavioral processes other than those being assayed experimentally. Additionally, Parkinson's disease, which includes dysfunction of the dopaminergic system, is associated with vocal performance and plasticity deficits (Ramig et al., 2008; Mollaei et al., 2013). However, because Parkinson's disease involves pathologies that extend beyond a simple loss of dopaminergic neurons (Lang and Obeso, 2004), it is difficult to use clinical studies to pinpoint DA's role in vocal behavior.

Songbirds provide a well-defined neural circuit in which to investigate DA's role in vocal learning. As in a number of forms of mammalian behavioral plasticity (Ogura et al., 2005; Eckart et al., 2010), the basal ganglia are crucial for vocal plasticity in songbirds. Area X (Fig. 1a,b), the song system's basal ganglia component, is a nucleus in the anterior forebrain pathway (AFP), a basal ganglia- thalamocortical loop long implicated in vocal learning (Sohrabji et al., 1990; Scharff and Nottebohm, 1991; Brainard and Doupe, 2000). Area X is necessary for song learning but not performance. Its destruction abolishes normal song learning in juveniles and degrades the adaptive modification (but not performance) of song in adults (Sohrabji et al., 1990; Scharff and Nottebohm, 1991; Fee and Goldberg, 2011; Ali et al., 2013). How- 


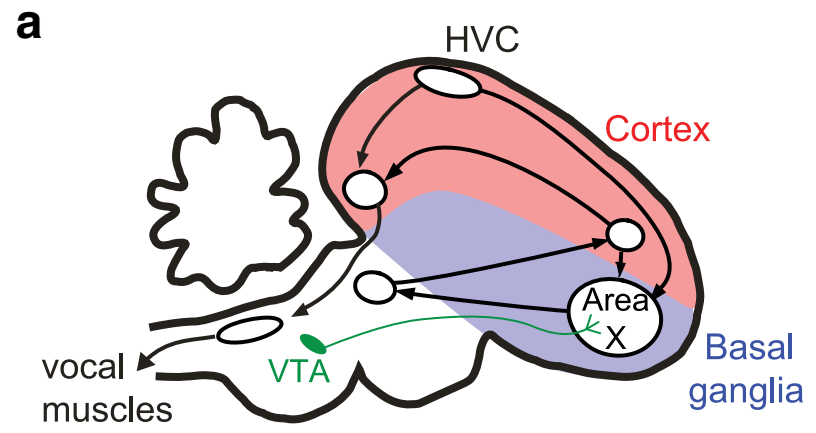

b

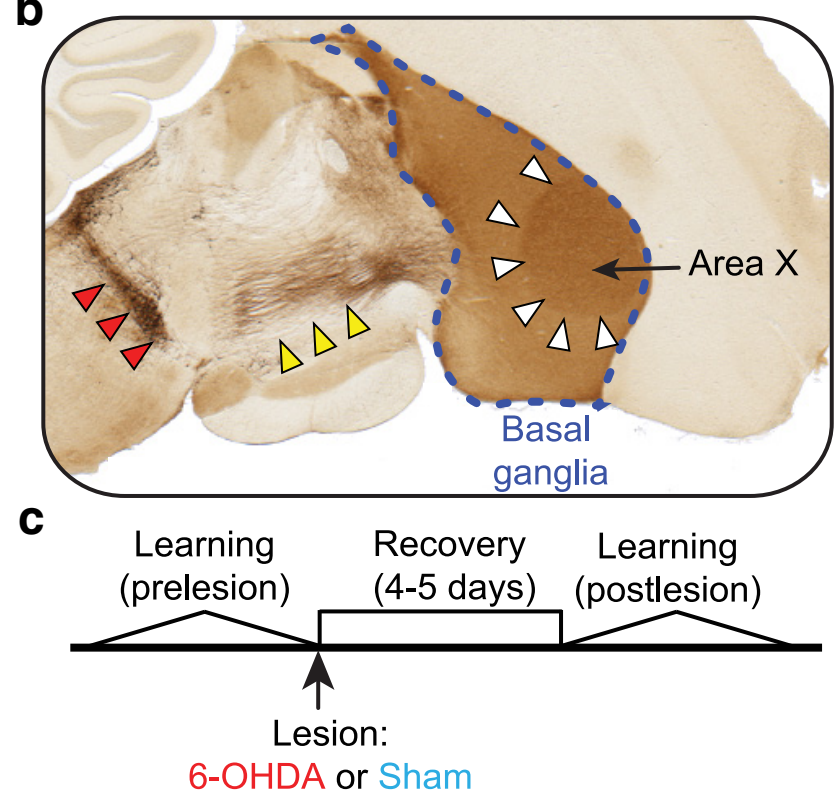

Figure 1. A song-specific basal ganglia nucleus receives strong dopaminergic input. $\boldsymbol{a}$, The song system includes area $X$, a basal ganglia nucleus critical for vocal learning. $\boldsymbol{b}$, A parasagittal section stained for $\mathrm{TH}$ shows heavy label within the basal ganglia (blue dotted line) with especially strong label in area $X$ (borders of $X$ indicated by white triangles). TH stain also shows dopaminergic cell bodies in the VTA/SNc (red triangles) and their ascending axons (yellow triangles). c, Experimental design (see Materials and Methods).

ever, it remains unclear how auditory error signals are conveyed to area X (Peh et al., 2015; Vallentin and Long, 2015). In mammals, dopaminergic inputs to the basal ganglia convey errorrelated signals, suggesting that dopaminergic afferents to area $\mathrm{X}$ might guide vocal learning in songbirds (Doya and Sejnowski, 1998; Graybiel, 2005; Turner and Desmurget, 2010; Fee and Goldberg, 2011; Leblois, 2013; Colombo, 2014). However, to our knowledge, no studies have directly addressed how DA contributes to vocal learning. We therefore reasoned that selectively lesioning dopaminergic inputs to area $\mathrm{X}$ would allow us to isolate DA's contribution to learning without inducing performance deficits.

We used the neurotoxin 6-hydroxydopamine (6-OHDA) to reduce dopaminergic innervation within area $\mathrm{X}$. In mammalian systems, 6-OHDA injections are commonly used to selectively eliminate dopaminergic fibers and cell bodies (Schober, 2004). The dopaminergic cells innervating area X originate in the VTA and $\mathrm{SNc}$ and are spatially intermingled with dopaminergic neurons that project to other parts of the striatum (Person et al., 2008), precluding injection directly into the VTA/SNc. Instead, we directly microinjected 6-OHDA into area $\mathrm{X}$ to reduce dopaminergic innervation of area $\mathrm{X}$, but not surrounding striatum, and quantified the resulting effects on song performance and vocal learning (Fig. 1c).

\section{Materials and Methods}

All subjects were adult ( $>100$-d-old) male Bengalese finches (Lonchura striata var. domestica). All procedures were approved by Emory University's Institutional Animal Care and Use Committee.

\section{Vocal learning paradigm and behavioral analysis}

Adaptive changes in the pitch (fundamental frequency) of targeted syllables were driven using a disruptive auditory stimulus as described previously (Tumer and Brainard, 2007). Briefly, when the pitch of a particular "targeted" syllable was above (or below) a particular threshold, a blast of white noise was played through the speakers, a contingency previously shown to induce birds to lower (or raise) vocal pitch to avoid white noise playback. During reinforcement, a randomly selected subset of target syllables (10\%) were selected as "catch trials" during which white noise was not played back, allowing quantification of holistic syllable features, such as sound amplitude. The frequency threshold for white noise was determined using the target syllable's pitch distribution from songs produced the morning of the first white noise day $(>25$ song bouts). To drive pitch down (or up), the targeting software was set to trigger a $40-50 \mathrm{~ms}$ white noise blast whenever target syllable pitch was above the 10th percentile (or below the 90th percentile) of this distribution.

All behavioral experiments began with a $3 \mathrm{~d}$ baseline period in which no white noise playbacks occurred. Postlesion baseline occurred during postsurgery days $4-6$ or $5-7$. In prelesion experiments, birds were exposed to white noise training for $3 \mathrm{~d}$. In postlesion experiments, training continued for at least $3 \mathrm{~d}$ plus up to 3 additional days to ensure that birds had sung at least $90 \%$ as many songs as during the prelesion white noise regimen, allowing comparison of learning between prelesion white noise day 3 and the approximately trial-matched postlesion white noise day. This extra training means that the white noise day immediately preceding washout day 1 is not necessarily white noise day 3 . Although across experiments lesions did not significantly affect the amount of song production on average (see Results), comparing trial-matched prelesion and postlesion days allows us to control for the effect of different amounts of vocal practice on the amount of learning in individual animals. Notably, as described in Results, all findings were qualitatively identical if prelesion and postlesion learning was compared on the same chronological day (white noise day 3) rather than the trial-matched day. Each bird's postlesion trial-matched day was the day where (by the end of the day) birds had sung the closest number of songs under the white noise regimen as they had after prelesion day 3 . Across birds postsham or postlesion, white noise training ended after days 3, 4, 4, 5 (for shams) and 2, 2, 3, 3, 6 (for 6-OHDA), and for each trial-matched day the total number of songs was within $10 \%$ of the number produced after prelesion day 3 . Daily targeting sensitivity (hit rate) had a median value of $92 \%$ across all experiments (range, 58\%-99\%). Daily targeting precision ( 1 - falsepositive rate) had a median value of $93 \%$ across all experiments (range, $51 \%-100 \%)$. Across experiments neither sensitivity nor precision was significantly different between the prelesion and postlesion epochs (Kolmogorov-Smirnov tests, $p>0.25$ ). All singing was undirected (i.e., in the absence of a female bird) throughout the white noise experiments.

After the last white noise day, we withdrew reinforcement and recorded song for 3 additional days to monitor spontaneous pitch restoration back to baseline, which typically occurs after several days (Tumer and Brainard, 2007; Warren et al., 2011). Throughout this paper, we refer to this time period as "washout" and the birds' process of returning vocal acoustics to their baseline values as "restoration." Washout occurred between 11 and $18 \mathrm{~d}$ after surgery, depending on the bird.

Although all singing was undirected (i.e., no female bird was present) during baseline, white noise training, and washout, we collected femaledirected songs from 4 birds (three pre- and post-6-OHDA lesion, one presham) to assess the effect of 6-OHDA lesion on social contextdependent changes in pitch variability (Sakata et al., 2008). We obtained directed songs 1-4 d after washout was concluded in both prelesion and postlesion conditions. Interleaved directed and undirected songs were 
Table 1. Injection parameters for 6-0HDA lesioned birds ${ }^{a}$

\begin{tabular}{|c|c|c|c|c|c|c|c|c|}
\hline Bird ID & Hemisphere & $\begin{array}{l}6-\mathrm{OHDA} \\
\text { concentration } \\
(\mathrm{mg} / \mathrm{ml})\end{array}$ & $\begin{array}{l}\text { Injected } \\
\text { volume (nl) }\end{array}$ & $\begin{array}{l}\text { Total 6-OHDA } \\
\text { injected }(\mu \mathrm{g})\end{array}$ & AP coordinates (mm) & ML coordinates $(\mathrm{mm})$ & $\begin{array}{l}\text { DV } \\
\text { coordinates }(\mathrm{mm})\end{array}$ & $\begin{array}{l}\text { Days killed } \\
\text { after surgery }\end{array}$ \\
\hline Bird 1 & Left & 8 & 179.4 & 1.435 & $5.1,5.5,5.9,6.3$ (4.8 for Med_X) & $0.9,1.55,2.2$ (0.9 for Med_X) & 3.18 (2.6 for Med_X) & 18 \\
\hline Bird 2 & Left & 8 & 124.2 & 0.994 & $5.3,5.8,6.3$ & $1.0,1.6,2.2$ & $3.1--1$ & 14 \\
\hline Bird 2 & Right & 8 & 124.2 & 0.994 & $5.3,5.8,6.3$ & $1.0,1.53,2.05$ & 3.4 & 14 \\
\hline Bird 3 & Left & 8 & 179.4 & 1.435 & $5.1,5.5,5.9,6.3$ (4.8 for Med_X) & $0.9,1.55,2.2$ (0.8 for Med_X) & 3.18 (2.6 for Med_X) & 21 \\
\hline Bird $4^{b}$ & Right & 8 & 179.4 & 1.435 & $5.1,5.5,5.9,6.3$ (4.8 for Med_X) & $0.9,1.55,2.2$ (0.8 for Med_X) & 3.18 (2.6 for Med_X) & 22 \\
\hline Bird 5 & Left & 8 & 124.2 & 0.994 & $5.1,5.7,6.3$ & $0.9,1.55,2.2$ & 3.18 & 14 \\
\hline Bird 5 & Right & 8 & 138 & 1.104 & 5.1, 5.7, 6.3 (4.8 for Med_X) & $0.9,1.55,2.2$ (0.8 for Med_X) & 3.18 (2.6 for Med_X) & 14 \\
\hline
\end{tabular}

${ }^{a} \mathrm{AP}$ and $\mathrm{ML}$ coordinates are on a grid such that each AP coordinate is paired with each ML coordinate in three or four rows. AP and ML coordinates are relative to $\mathrm{Y}_{0}$, and $\mathrm{ML}$ coordinates are offset to the left or right of $\mathrm{Y}_{0}$ depending on the hemisphere. DV coordinates are relative to the exposed brain surface. "Med_X" indicates a single extra injection that was intended (but not conclusively proven) to hit the medial-most portion of area X, where its pear shape comes to a dorsal and posterior point.

${ }^{b}$ In two additional birds, we performed unilateral 6-OHDA lesions and TH/NeuN stains to compare area X cell counts in sham- and 6-OHDA-lesioned hemispheres (see Materials and Methods). In these birds, we used identical injection coordinates and volumes as Bird 4.

collected as described previously (Sakata et al., 2008). We obtained 1207 directed (919 undirected) syllable iterations across 11 prelesion syllables and 707 directed ( 688 undirected) iterations across 8 postlesion syllables and analyzed an equal number of interleaved undirected songs per bird/ condition ( $>30$ syllable iterations per syllable and condition).

Custom-written MATLAB software (The MathWorks) was used for data analysis. Pitch changes were quantified in units of semitones as follows:

$$
s=12 * \log _{2}(h / b)
$$

where $s$ is the pitch change (in semitones) of the syllable, $h$ is the pitch (in Hertz) of the syllable, and $b$ is the average baseline pitch (in Hertz) of the syllable. On each baseline, white noise day, and washout day, we quantified the pitch, amplitude, and spectral entropy of the targeted syllable in 100 song bouts spaced evenly throughout the day (or all songs when birds sang $<100$ songs on a given day), as described previously (Sober et al., 2008). To assess lesion-related changes in the quantity of song produced, for each bird we quantified the ratio of the mean number of song bouts per day after 6-OHDA or sham lesion to the mean number produced per day before lesion. We then tested whether the distribution of ratios from 6-OHDA-injected animals differed from both unity and the distribution of ratios from the sham-injected group using one- and two-sample Kolmogorov-Smirnov tests, respectively. These tests allow us to quantify whether neurotoxin injection had a consistent effect on the amount of song production relative to both preinjection behavior and any effects of sham injections.

To assess changes in vocal pitch during the washout period (i.e., after the cessation of white noise training), we fit an exponential decay model to the pitch data as follows:

$$
p(t)=p_{\text {initial }} e^{-\frac{t}{\tau}}+p_{\text {final }}\left(1-e^{\left.-\frac{t}{\tau}\right)}\right.
$$

where $p_{\text {initial }}$ was set to the mean pitch on the final day of $\mathrm{WN}$ training, and fit parameters were $\tau$ and $p_{\text {final }}$, corresponding to the time constant of pitch restoration during washout and the asymptote of the exponential fit (final value of pitch if restoration were to reach equilibrium), respectively. We note that this exponential model is a generalization of a model we have used previously to quantify the time course of learning when songbirds experience real-time errors in the pitch of auditory feedback delivered via miniature headphones (Sober and Brainard, 2012); in that earlier model, $p_{\text {initial }}$ was zero, because changes in vocal pitch were quantified relative to baseline error of zero.

\section{6-OHDA and sham lesions}

Subjects were randomly assigned to either the sham or 6-OHDA lesion group. Before injections, birds were anesthetized with ketamine, midazolam, and isoflurane, mounted in a stereotax at a $20^{\circ}$ beak angle relative to the table surface, and small craniotomies were made above area $\mathrm{X}$.
Table 2. Injection parameters for sham-lesioned birds ${ }^{a}$

\begin{tabular}{llll}
\hline Bird ID & Hemisphere & Injection parameters & $\begin{array}{l}\text { Days killed } \\
\text { after surgery }\end{array}$ \\
\hline Bird 6 & Left & Identical to Bird 5 but no 6-OHDA & 17 \\
Bird 6 & Right & Identical to Bird 5 but no 6-OHDA & 17 \\
Bird 7 & Left & Identical to Bird 2 but no 6-OHDA & 18 \\
Bird 7 & Right & Identical to Bird 2 but no 6-OHDA & 18 \\
Bird 8 & Left & Identical to Bird 1 but no 6-OHDA & 27 \\
Bird 8 & Right & Identical to Bird 1 but no 6-OHDA & 27 \\
Bird 9 & Left & Identical to Bird 3 but no 6-OHDA & 23 \\
Bird 9 & Right & Identical to Bird 3 but no 6-OHDA & 23 \\
\hline
\end{tabular}

${ }^{a}$ Each sham-lesioned bird's injection parameters were precisely matched to those of a 6-OHDA bird for consistency. "no 6-OHDA" indicates that birds were injected with saline and ascorbic acid (instead of 6-OHDA, saline, and ascorbic acid).

Lesioned birds received bilateral injections of $11.8 \mathrm{mg} 6-\mathrm{OHDA}-\mathrm{HBr} / \mathrm{ml}$ (i.e., $8 \mathrm{mg}$ freebase $6-\mathrm{OHDA} / \mathrm{ml}$ ) and $2 \mathrm{mg}$ ascorbic acid/ml (stabilizer) in a $0.9 \% \mathrm{NaCl}$ solution into area $\mathrm{X}$ using a Drummond Scientific Nanoject II auto-nanoliter injector. During each injection, the pipet was lowered into the brain along a plane perpendicular to the table surface. We injected $13.8 \mathrm{nl}$ at each location and waited $30 \mathrm{~s}$ before raising the pipet. For sham lesions, only vehicle ( $2 \mathrm{mg}$ ascorbic acid $/ \mathrm{ml}$ in $0.9 \% \mathrm{NaCl}$ ) was injected per the procedure described above. All birds recovered from surgery within a few hours and usually sang the next day.

We varied injection coordinates and volumes slightly between birds and hemispheres to optimize injection parameters. For detailed parameters for each bird, see Tables 1 and 2. Total injection volume in each hemisphere ranged from 124.2 to $179.4 \mathrm{nl}$ for all hemispheres, except one $(234.6 \mathrm{nl})$. Necrotic damage within area X was observed in only one hemisphere of one bird (the right hemisphere of Bird 3, which was the hemisphere receiving the largest total injection volume of $234.6 \mathrm{nl}$ ). This necrotic damage affected $8 \%$ of the total volume of area $\mathrm{X}$ in the affected hemisphere. As our results are unaffected by removing Bird 3 from our dataset, we have included it in our analysis. As described below in Histology, we also performed a number of other analyses to investigate whether 6-OHDA injections killed neurons in area $\mathrm{X}$ in cases where no necrosis was apparent.

To cover the greatest possible volume of area $\mathrm{X}$ while still injecting low volumes, we placed injections ( $13.8 \mathrm{nl}$ each) on a $3 \times 3,3 \times 4$, or $4 \times 4$ grid. Each grid was located at a single dorsal-ventral (DV) coordinate between 3.1 and $3.4 \mathrm{~mm}$ and individual injections were evenly spaced anterior-posterior (AP) and medial-lateral (ML) coordinates between 5.1 and $6.3 \mathrm{~mm}$ and $0.9-2.2 \mathrm{~mm}$, respectively. All AP and ML coordinates were relative to the posterior edge of a Y-shaped sinus visible beneath the inner skull layer, whereas DV coordinates were relative to the exposed brain surface. In three birds (and right hemisphere of a fourth), there was an additional injection outside of the main grid intended to hit 
a

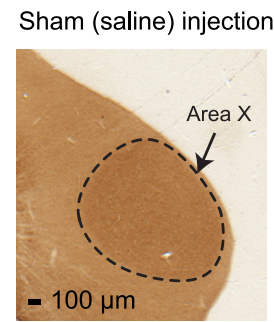

d

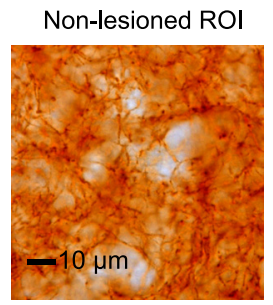

6-OHDA injection

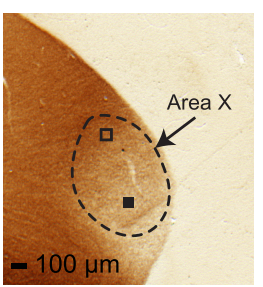

Lesioned ROI

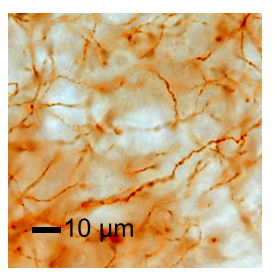

b

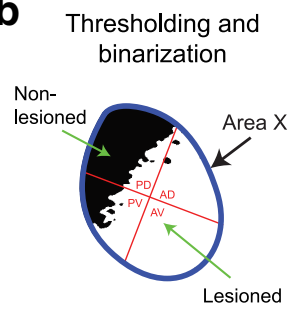

e

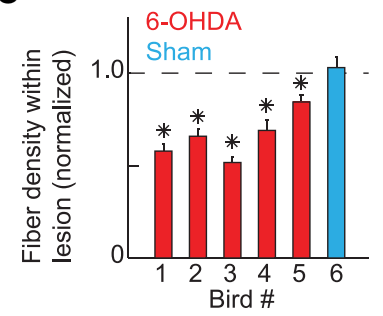

C

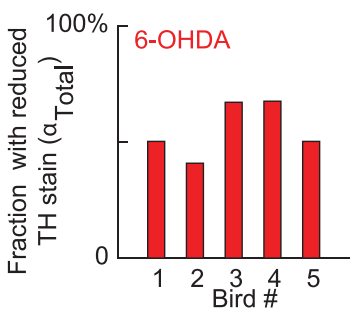

Figure 2. Lesions of dopaminergic inputs to area X. a, Comparison of TH stain in sham (left) and 6-OHDA-lesioned (right) brains shows a reduction in the OD of stain in 6-OHDA-injected animals. $\boldsymbol{b}$, To measure the loss of dopaminergic inputs, we used an OD threshold to divide images of area Xinto "lesioned" (white) and "nonlesioned" (black) subregions. Additionally, to quantify the location of lesions, we divided area Xinto dorsal, ventral, anterior, and posterior subregions. Red lines and letters indicate subregions that are both posterior and dorsal (PD), anterior and dorsal (AD), posterior and ventral (PV), and anterior and ventral (AV). Because all sections were cut parasagittally, medial and lateral subregions were designated by categorizing each section as belonging to either the medial or lateral half of area X. $\boldsymbol{c}$, Using the binarization shown in $\boldsymbol{b}$, we quantified the fraction of area X in which TH stain was reduced ( $\alpha_{\text {Totali }}$; 5 ee Materials and Methods). $\boldsymbol{d}$, We also quantified the density of TH-positive fibers both within and outside the lesioned subregion of area X ("lesioned ROI" and "nonlesioned ROI," respectively) in individual histological sections. Examples of lesioned and nonlesioned ROIs are shown as filled and empty squares in $\boldsymbol{a}$, respectively. $\boldsymbol{e}$, Within each section, we normalized the fiber density in the lesioned ROl by the density in the nonlesioned ROI from the same image. Histogram and error bars indicate the mean \pm SEM of this measure across five 6-OHDA-injected birds and one sham. ${ }^{*} p<0.05$ (two-sided Kolmogorov-Smirnov tests). We obtained the same result when raw (un-normalized) fiber density measures were used.

the most medial portion of area $\mathrm{X}$, where its pear shape comes to a dorsal and posterior point at coordinates $\mathrm{DV}=2.6 \mathrm{~mm}, \mathrm{AP}=4.8 \mathrm{~mm}$, and $\mathrm{ML}=0.8-0.9 \mathrm{~mm}$.

\section{Histology}

Each bird was perfused 14-23 d after 6-OHDA or sham lesion. Dissected brains were fixed overnight at $4^{\circ} \mathrm{C}$ in $4 \%$ formaldehyde, sunk in $30 \%$ sucrose for 1-4 d, and sliced in $40 \mu \mathrm{m}$ sections on a microtome. We performed chromogenic TH stains on odd-numbered sections (to assess loss of area X catecholaminergic fiber innervation) and either Nissl stains (7 birds) or fluorescent NeuN and fluorescent TH stains (2 birds; one sham, one 6-OHDA) on even-numbered sections (to assess postlesion necrosis).

In two additional birds, we performed unilateral 6-OHDA lesions (one bird in left and one in right hemisphere) and perfused $11 \mathrm{~d}$ after surgery. We performed chromogenic tyrosine hydroxylase (TH) and fluorescent NeuN stains on alternating sections to compare area $\mathrm{X}$ cell counts in sham- and 6-OHDA-lesioned hemispheres together with the two bilaterally lesioned NeuN-stained birds mentioned above. All hemispheres in these birds used the same injection coordinates and volumes as Bird 4 in Table 1 . These birds were housed singly after surgery (no other birds were present), and no behavioral data were collected from them.

For chromogenic TH immunohistochemistry, all steps used $0.2 \mathrm{M}$ PBS ( 23 g sodium phosphate (dibasic) +5.25 g sodium phosphate (monobasic) per $1 \mathrm{~L}$ deionized $\mathrm{H}_{2} \mathrm{O}$ ) as the solvent unless otherwise indicated. Between each of the following steps, tissue was rinsed three times for 10 $\min$ in $0.2 \mathrm{M}$ PBS. Tissue was first incubated in $0.3 \% \mathrm{H}_{2} \mathrm{O}_{2}$ for $30 \mathrm{~min}$ and $1 \% \mathrm{NaBH}_{4-}$ for $20 \mathrm{~min}$. It was then incubated at room temperature overnight in a solution containing primary antibody against tyrosine hydroxylase (Millipore MAB318; 1:4000), 0.3\% Triton X-100, and 5\% normal horse serum. Tissue was then incubated in biotinylated antimouse secondary antibody (Vector Laboratories horse anti-mouse; 1:200 $+0.3 \%$ Triton X-100) for $1 \mathrm{~h}$, followed by $1 \mathrm{~h}$ in avidin-biotin-complex (ABC) solution (Vector Laboratories Vectastain ABC kit; 1\% HRPconjugated streptavidin, $1 \%$ biotin, $0.3 \%$ Triton X-100, and $20 \mathrm{mg} \mathrm{NaCl} /$ $\mathrm{ml}$ ). ABC solution was left to react for $30 \mathrm{~min}$ before use. Finally, tissue was exposed to diaminobenzidine (DAB) solution for exactly $5 \mathrm{~min}$. Per tray, DAB solution contained two DAB tablets (Amresco E733; $5 \mathrm{mg}$ $\mathrm{DAB}$ per tablet) in $20 \mathrm{ml}$ of purified water.

For fluorescent immunohistochemistry, tissue was incubated in $1 \%$ $\mathrm{NaBH}_{4}$ for $20 \mathrm{~min}$, then blocked in 5\% normal horse serum and $0.5 \%$ Triton X-100. Tissue was incubated for $48 \mathrm{~h}$ at $4^{\circ} \mathrm{C}$ in primary antibodies against tyrosine hydroxylase (Millipore MAB318; 1:2000) and NeuN conjugated to AlexaFluor488 (Millipore ABN78A4; 1:2000) in 1\% normal horse serum and $0.5 \%$ Triton X-100. Tissue was then rinsed and incubated in biotinylated anti-mouse secondary (Vector Labs BA-2000; $1: 200+0.5 \%$ Triton $\mathrm{X}-100)$ for $1 \mathrm{~h}$ at room temperature, rinsed, and incubated in streptavidin-AMCA (SA-5008; $5 \mu \mathrm{g} / \mathrm{ml}+0.5 \%$ Triton $\mathrm{X}-100)$ for $1 \mathrm{~h}$ at room temperature. Sections were mounted and coverslipped with Fluoro Gel with 1,4-diazabicyclooctane. Similar to chromogenic immunohistochemistry, $0.2 \mathrm{M}$ PBS was used as a solvent, and tissue was rinsed three times for $10 \mathrm{~min}$ after each step, except for blocking.

\section{Image analysis}

Lesion size and location. We quantified both the fraction of area $\mathrm{X}$ that exhibited reduced TH label and the extent to which the lesions affected different subregions of area $\mathrm{X}$ by measuring lesion-induced changes in the density of TH label. Images were acquired on a slide scanner (Meyer Instruments PathScan Enabler IV; 24 bit color, 7200 dpi, "sharpen more" filter, brightness, and contrast level 50). A custom-written ImageJ (version 1.47) macro was used to manually outline area $\mathrm{X}$ as an ROI on each TH-stained section.

As shown in Figure $2 b$, in each image an optical density (OD) threshold was established and then used to binarize the image so that each pixel within area X was categorized as belonging to either the "lesioned" (indicated by a lighter TH stain in that area) or "nonlesioned" subregion of area X. We use the terms "lesioned" and "nonlesioned" to differentiate subregions of area $\mathrm{X}$ in 6-OHDA-injected birds that do or do not exhibit a loss of TH label (i.e., these terms do not refer to 6-OHDA injected vs sham-lesioned animals). Because the level of background staining varied somewhat across sections, the OD threshold was set manually. We then used the binarized images to quantify the fraction of area $\mathrm{X}$ in that image that had been lesioned as follows: 


$$
\alpha_{i}=\frac{N_{i}^{\text {lesioned }}}{N_{i}^{\text {lesioned }}+N_{i}^{\text {non-lesioned }}}
$$

Where $\alpha_{i}$ is the fraction of area X affected by the lesion in histological section $i$. Terms $N_{i}^{\text {lesioned }}$ and $N_{i}^{\text {non-lesioned }}$ represent the number of lesioned and nonlesioned pixels in the image, respectively.

To assess the total fraction of $\mathrm{X}$ that received lesions in a given bird $\left(\alpha_{\text {Total }}\right)$, we quantified the following:

$$
\alpha_{\text {Total }}=\frac{\sum_{i=1}^{k} N_{i}^{\text {lesioned }}}{\sum_{i=1}^{k}\left(N_{i}^{\text {lesioned }}+N_{i}^{\text {non-lesioned }}\right)}
$$

where the lesioned and nonlesioned pixels are summed across the $k$ sections of area $\mathrm{X}$. This measure is summed across the two hemispheres, resulting in a single value of $\alpha_{\text {Total }}$ for each bird which was then compared with each bird's learning behavior (see Relationship between lesion size/ location and behavioral data). Additionally, we quantified the fraction of lesion in subregions of area $\mathrm{X}$. To do so, we divided images of area $\mathrm{X}$ into dorsal, ventral, anterior, posterior, lateral, and medial subregions, as shown in Fig. $2 b$. Thus, each of the six subregions comprised half of area $\mathrm{X}$ (e.g., the dorsal subregions included measurements from the dorsal half of area $\mathrm{X}$ in each section). We then calculated the fraction of each subregion that was lesioned ( $\alpha_{\text {dorsal }}, \alpha_{\text {ventral }}$, etc.) using the procedure described above.

Alternate analysis of $O D$. In addition to the above analysis of lesion size and location, we performed an alternate analysis that did not rely on manually establishing an OD threshold. A custom-written ImageJ macro was used to manually outline area $\mathrm{X}$ as an ROI on each TH-stained section and place $0.5-\mathrm{mm}$-diameter ROI circles on representative areas of cortex (just dorsal to area $\mathrm{X}$ ) and non-X-striatum (just posterior to area $\mathrm{X}$ near the dorsal border of the striatum). In some cases (e.g., Fig. $2 a$, right) loss of TH label extended slightly outside of the border of area X; the "non-X-striatum" ROI was positioned to exclude such areas. OD was quantified by converting the image to 8 bit grayscale and then measuring the average pixel value in each ROI.

To assess the effects of 6-OHDA injection into area $\mathrm{X}$, we quantified $\frac{O D_{\text {Area }} \mathrm{X}}{\mathrm{OD}_{\text {Striatum }}}$, the ratio of OD in the area X ROI to the non-X-striatum ROI. This ratio was calculated separately in each TH-stained section to account for cross-section and cross-animal variations in stain density. We also performed an alternate analysis in which the OD of the cortex ROI was treated as background signal, and OD ratio was computed as $\left(\frac{O D_{\text {Area }}-O D_{\text {cortex }}}{O D_{\text {Striatum }}-O_{\text {cortex }}}\right)$ within each stained section. This alternate technique yielded nearly identical results as the primary analysis. Quantifying the distribution of OD ratios in sham-lesioned animals (which is typically $>1$ because area $X$ receives denser catecholaminergic input than the surrounding striatum) (Soha et al., 1996) allowed us to determine the 95\% confidence interval of this metric in sham-lesioned brains. Any section from a 6-OHDA-injected animal with an OD ratio beyond the $95 \%$ interval therefore exhibits a significant reduction in TH staining density with $p<0.05$.

Neuron counts. We quantified the number of surviving neurons following 6-OHDA (and sham) lesions in area X as well as in VTA/SNc, which sends a massive dopaminergic projection to the striatum, and in the locus ceruleus (LC), which may send a weak noradrenergic projection to area X (but see Mello et al., 1998; Castelino et al., 2007). We quantified the number of neurons in area $\mathrm{X}$ by imaging $4-10$ sections from each of the four NeuN-stained brains (see Histology) at $40 \times$ magnification $(0.75$ zoom) using a Leica SP8 multiphoton microscope. We excluded 3 of the 29 images due to an imaging artifact. Image was used to convert images to 8 bit grayscale, threshold based on pixel intensity to create a binary image, reduce noise (Remove Outliers in ImageJ), and separate touching cell bodies (Watershed in ImageJ). Cell bodies were counted using the Analyze Particles plug-in. Identical acquisition and processing parameters were used for all images.

To assess whether the number of NeuN-stained cells in area X differed between experimental conditions (6-OHDA vs sham), we performed a multilinear regression analysis with birds and lesion condition as factors. Including each bird as a factor in the model increased our power by controlling for any between-bird differences. We combined data across NeuN-stained birds (one bilateral sham, one bilateral 6-OHDA, two unilateral 6-OHDA as described in Histology) and fit a standard multiple linear regression model (Kutner et al., 2005) as follows:

$$
\mathrm{y}_{\mathrm{ij}}=\beta_{0}+\beta_{1} * \mathrm{~b} 2_{\mathrm{ij}}+\beta_{2} * \mathrm{~b} 3_{\mathrm{ij}}+\beta_{3} * \mathrm{~b} 4_{\mathrm{ij}}+\beta_{4} * \mathrm{C}_{\mathrm{ij}}+\varepsilon_{\mathrm{ij}}
$$

where $y_{i j}=$ cell counts for bird $\mathrm{i}$ and image $\mathrm{j}, \mathrm{C}_{\mathrm{ij}}$ is an indicator variable to represent experimental condition $\left(\mathrm{C}_{\mathrm{ij}}=1\right.$ if image ij is from a 6-OHDA hemisphere, 0 if from a sham hemisphere), b2-b4 are indicator variables to represent bird-specific effects $\left(\mathrm{b} 2_{\mathrm{ij}}=1\right.$ if image ij is from bird 2, 0 otherwise), $\beta$ values are regression coefficients, and $\varepsilon$ is the residual error, assumed to be normally distributed. The term $\beta_{4} * \mathrm{C}$ represents the condition-specific effect after controlling for bird-specific effects $\left(\beta_{0}\right.$ $\left.+\beta_{1} b_{2}+\beta_{2} b_{3}+\beta_{3} b_{4}\right)$. Because indicator variables are 1 or 0 depending on the bird, the term $\beta_{0}$ represents the effect of Bird 1. To determine whether 6-OHDA-lesioned hemispheres had fewer cells in area $\mathrm{X}$ than sham-lesioned hemispheres, we performed a partial $F$ test on whether $\beta_{4}$ is significantly different from zero after including the other factors.

To quantify neuron numbers in VTA/SNc and LC in each white noise trained bird, chromogenically TH-stained sections were imaged at $10 \times$ on an Olympus IX51 Widefield microscope with a Hamamatsu Orca ER CCD camera (for VTA/SNc) or an Axioplan widefield microscope with an Optronics camera (for LC). Ten sections containing VTA/SNc and two containing LC were imaged for each subject. Because sections were cut parasagittally, we did not attempt to identify the border between VTA and SNc. The most medial sections of VTA and the most lateral sections of $\mathrm{SNc}$ were not imaged because these regions contain few area $\mathrm{X}$-projecting neurons (Person et al., 2008). Image acquisition parameters were held constant across subjects. In cases where the region being imaged was too large to fit into a single field of view, multiple images were taken and stitched together using the ImageJ Pairwise Stitching plug-in (Preibisch et al., 2009).

VTA/SNc and LC cell counts were performed using the ImageJ Cell Counter plug-in by four raters who were blinded to bird identity and treatment condition. Rater bias was quantified by having all raters count cells in the same histological sections and comparing mean counts across raters. The mean count from each individual was used to linearly scale all counts from that rater, with all correction terms having an absolute value of $\leq 13 \%$. Cell count results were qualitatively identical even if this correction term was not applied. As described in Results, cell counts from all birds revealed no significant difference in either VTA/SNc or LC. However, a post hoc power analysis revealed that we would be unlikely to detect such change given the very small size of our neurotoxin injections, given that area $\mathrm{X}$ comprises $\sim 10 \%$ of the total volume of the basal ganglia (Karten et al., 2013) and that our lesions affected only part of area X. To perform the power analysis, we made two extremely conservative assumptions to put an upper bound on the number of catecholaminergic neurons that project to area $\mathrm{X}$. First, we can assume that area $\mathrm{X}$ received $10 \%$ of the catecholaminergic input (the actual fraction is likely much lower given that both VTA and LC send inputs to the forebrain in addition to the striatum). Second, we can assume that 6-OHDA injections lesion will kill $100 \%$ of neurons that project to the affected region of the striatum (the actual fraction of neurons killed is likely significantly lower than this). Therefore, given that our lesions affected $\sim 50 \%$ of area $X$, we would expect that our lesions would kill at most $5 \%$ of catecholaminergic neurons projecting to area $\mathrm{X}(50 \% \times 10 \%)$, and likely much less.

We therefore performed a power analysis to quantify whether we would be likely to be able to detect a $5 \%$ change in neuron number. Across repeated measurements of the same TH-stained section, our cell counts had an SD of $\sigma_{\text {section }}=10 \%$ relative to the mean. We assessed the total number of $\mathrm{TH}^{+}$cells in the VTA by summing cell counts across $n_{\text {sections }}=10$ histological sections. Assuming that cell counts of different sections represent independent measurements, we therefore expect that the total cell count for each bird has a SD of the following: 


$$
\left.\sigma_{\text {Bird }}=\sqrt{n_{\text {sections }} \sigma_{\text {section }}^{2}}=32 \% \text { (relative to the mean }\right)
$$

We then quantified the power of an analysis to detect a 5\% difference in the number of $\mathrm{TH}^{+}$neurons with a SD of $32 \%$ and a total of 5 measurements ( 5 birds per group). This analysis yielded a power of 0.07 , indicating that we would only have a $7 \%$ chance of detecting such a difference. Our failure to detect a significant change in $\mathrm{TH}^{+}$cell body number (see Results) is therefore unsurprising given the very small size of our neurotoxin injections within the basal ganglia.

Analysis of fiber density. For TH fiber density analysis in area X, sections were imaged at $63 \times$ using a Zeiss Axioplan 2 Widefield microscope with an AxioCam HRc Color Camera. Lesioned and nonlesioned portions of area $\mathrm{X}$ within a single section were selected based on previously captured images (see Lesion size and location). Image acquisition parameters varied slightly between sections but were held constant for lesionnonlesioned pairs within a single section.

Mammalian studies frequently induce unilateral lesions (e.g., injecting 6-OHDA into the striatum on one side and vehicle into the other side), allowing the experimenter to normalize the fiber density in the lesioned hemisphere to that in the opposite hemisphere to compensate for stochastic variations in TH stain intensity (i.e., animal-by-animal variation that is unrelated to the experimental condition). Because all birds used in our behavioral experiments received bilateral lesions of dopaminergic inputs to area $\mathrm{X}$, we were unable to take this approach. Instead, we normalized the fiber density within the lesioned subregion of area $\mathrm{X}$ to the fiber density within a nonlesioned subregion in the same histological section, as described below. However, as described in the main text, we obtained qualitatively identical results when we did not perform this within-section density normalization.

Images were analyzed using ImageJ, with identical image-processing steps applied to every section. In each brain, we chose 10 tissue sections (five from each hemisphere) that contained both lesioned and nonlesioned subregions of area X. We then captured two images from each section, one from the lesioned and one from the nonlesioned subregion, and converted all images to 8 bit grayscale. To isolate TH-positive fibers, images were then bandpassed (FFT Bandpass Filter in ImageJ) to emphasize features with high spatial frequency (i.e., labeled axons) and then thresholded based on pixel intensity to create a binary image in which black pixels represented TH-stained fibers. After removing outlier pixels (Remove Outliers in ImageJ), we then measured the density of THpositive fibers by quantifying number of black pixels as a fraction of total pixels in the image. Fiber density from each lesioned subregion was then normalized to the density of the nonlesioned subregion in the same images. To assess the level of variation in this measure in a sham-lesioned bird, in one sham bird we randomly selected 5 of 10 ROIs to serve as the "lesioned" subregions.

\section{High performance liquid chromatography (HPLC)}

In a separate set of adult ( $>100$-d-old) male Bengalese finches $(n=6)$, we performed unilateral 6-OHDA lesions and used HPLC to compare area X DA and norepinephrine (NE) levels in lesioned and shamlesioned hemispheres. Each bird received a 6-OHDA lesion in area X in one hemisphere and a sham lesion in the other hemisphere using the same procedure described in 6-OHDA and sham lesions. All hemispheres across birds used the same injection coordinates and volumes as Bird 4 in Table 1. We alternated which hemisphere was injected with 6-OHDA, so half the birds received lesions in the left and half in the right hemisphere. The birds were housed singly after surgery (no other birds were present), and we did not collect any behavioral data from these animals.

Fourteen days after surgery, we decapitated each bird, rapidly harvested the brains, flash-froze them in powdered dry ice 2-4 min after decapitation, and stored them at $-80^{\circ} \mathrm{C}$. Frozen brains were sliced into $300 \mu \mathrm{m}$ parasagittal sections in a $-12^{\circ} \mathrm{C}$ cryostat, placed on slides, briefly wet-thawed to room temperature $(<20 \mathrm{~s})$ to allow tissue to settle on the slide and placed on dry ice. In each hemisphere, we made 1-mmdiameter, 300- $\mu \mathrm{m}$-thick circular tissue punches of area $\mathrm{X}$ in two sections using a previously described technique (Palkovits, 1973), placed both punches in a tube while still frozen and stored the sample at $-80^{\circ} \mathrm{C}$ until tissue was analyzed for monoamine content. Area X was identified by observing the frozen and briefly wet-thawed sections with the naked eye and through a dissecting microscope using a bright light and dark surface to enhance contrast.

NE and DA concentrations were determined by HPLC with coulometric detection using established methods (Pozdeyev et al., 2008). Each sample was processed individually (one sample per hemisphere). Briefly, samples were first homogenized in $0.1 \mathrm{~N} \mathrm{HClO}_{4}$ solution (containing $0.01 \%$ sodium metabisulfite and $25 \mathrm{ng} / \mathrm{ml}$ internal standard 3 , 4-dihydroxybenzylamine $\mathrm{HBr}$ ), and centrifuged at 13,000 $\times \mathrm{g}$ for $15 \mathrm{~min}$ at $4^{\circ} \mathrm{C}$. Supernatant fraction aliquots were injected into an Ultrasphere 5 $\mu \mathrm{m}$ ODS column, $250 \times 4.6 \mathrm{~mm}$ (Hichrom) and separated with a mobile phase containing $0.1 \mathrm{~m}$ sodium phosphate, $0.1 \mathrm{~mm}$ EDTA, $0.35 \mathrm{~mm}$ sodium octyl sulfate, and $7 \%(\mathrm{v} / \mathrm{v})$ acetonitrile, $\mathrm{pH}$ 3.2. DA and NE amounts (ng/sample) were then quantified by comparison with internal standards, with a standard curve generated with $0.1-5 \mathrm{ng}$ for each analyte. Protein ( $\mathrm{mg} / \mathrm{sample}$ ) was determined using the Lowry protein assay with a standard curve generated with 0-95 $\mu \mathrm{g}$ BSA (Lowry et al., 1951).

\section{Relationship between lesion size/location and behavioral data}

We used a stepwise regression procedure (Draper and Smith, 1998) to ask whether the magnitude and/or location of the loss of dopaminergic inputs to area $\mathrm{X}$ was predictive of the observed changes in learning behavior. To do this, we calculated the change in the absolute magnitude of learning due to 6-OHDA lesion as $\Delta_{\text {Absolute }}=\mu_{\text {post }}-\mu_{\text {pre }}$, where $\mu_{\text {pre }}$ and $\mu_{\text {post }}$ are the magnitude of pitch change before and after lesion, respectively, on last prelesion white noise day and trial-matched postlesion day. We then asked which of seven measurements of lesion size and location $\left(\alpha_{\text {Total }}, \alpha_{\text {dorsal }}, \alpha_{\text {ventral }}, \alpha_{\text {anterior }}, \alpha_{\text {posterior }}, \alpha_{\text {lateral }}, \alpha_{\text {medial }}\right)$ were significantly predictive of $\Delta_{\text {Absolute }}$. Stepwise regression analysis provides a systematic method for testing, which predictor terms should be included in a multilinear model by beginning with an initial model and testing changes in the model's predictive power that result from including or excluding individual predictor terms. We therefore applied this procedure to ask which, if any, of the seven candidate predictors should be included in a multilinear model that predicts $\Delta_{\text {Absolute }}$. This analysis concludes when neither including nor excluding any additional terms significantly improves the model ( $p<0.05$ after Bonferroni correction). In an alternate analysis, we used the same seven candidate predictors to produce a model

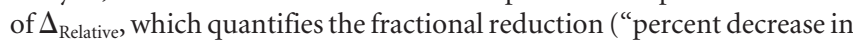
learning”) in learning behavior after lesion:

$$
\Delta_{\text {Relative }}=\left(1-\frac{\mu_{\text {post }}}{\mu_{\text {pre }}}\right) \times 100 \%
$$

In one 6-OHDA-lesioned bird, in the postlesion experiment, the bird made a small pitch change in the antiadaptive direction (i.e., $\mu_{\text {post }}$ was

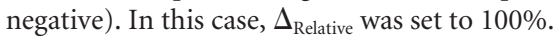

\section{Results}

We injected 6-OHDA into area X of adult male Bengalese finches, measured the ensuing effects on song performance and vocal learning, and quantified the lesion-induced depletion of area X's dopaminergic input. Following previous studies in mammals, we quantified dopaminergic innervation using an immunohistochemical stain for $\mathrm{TH}$, the rate-limiting enzyme in the DA synthesis pathway (Fig. 1b). As shown in Figure 2a, 6-OHDA injections substantially reduced $\mathrm{TH}$ label within area $\mathrm{X}$. To quantify the volume of area X affected by the lesion, we manually set an OD threshold for each image (see Materials and Methods; and Fig. $2 b$ ) and counted the fraction of pixels in which TH density fell below the threshold. As shown in Figure $2 c$, by this metric TH stain was reduced in $46 \%-68 \%$ of area $\mathrm{X}$ across birds, indicating that our lesions affected approximately half of the volume of the nucleus. Furthermore, to obtain a more direct measure of the lesions' effects on dopaminergic innervations, we analyzed tissue at high magnification to quantify the prevalence of TH-positive axons within area X (Fig. 2d). We found that 6-OHDA injections 


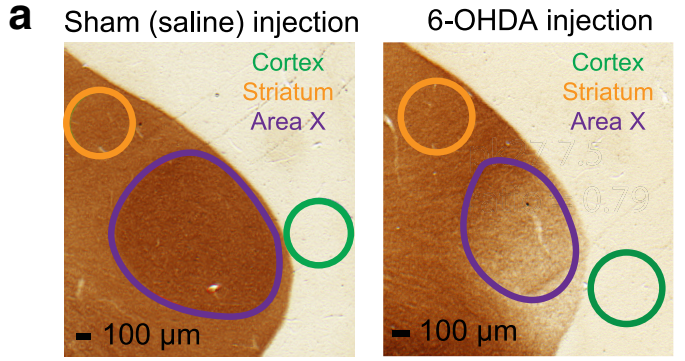

b

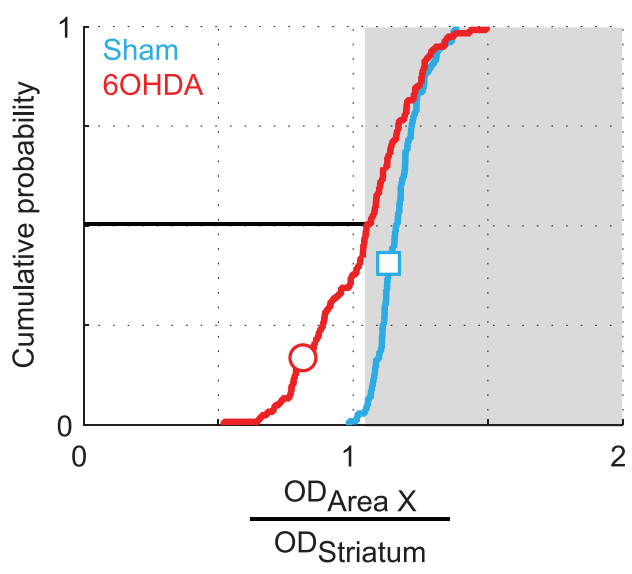

Figure 3. Alternate method of quantifying loss of TH label. In our primary analysis of lesion size (see Materials and Methods; Fig. 2b), we manually set an OD threshold to quantify the fraction of area $X$ in which $T H$ stain was reduced in each histological section. Here we present an alternate analysis (see Alternate analysis of OD) that does not rely on a within-image threshold but rather simply measured the mean $\mathrm{OD}$ across all of area X in each section. $\boldsymbol{a}$, Comparison of $\mathrm{TH}$ stain in sham (left) and 6-OHDA-lesioned (right) brains, showing the same sections as in Figure $2 a$. To measure the loss of dopaminergic inputs, in each histological section, we quantified the OD of TH stain across all of area $X$ (purple) in the adjacent striatum (orange). $\boldsymbol{b}$, Analysis of the ratio of $O D$ in area $X$ to that in striatum. In nearly all sections from sham-lesioned birds, $T H$ stain is darker in area $X$ than in surrounding striatum (OD ratio $>1$ ). In 6-OHDA-injected birds (red trace), $50 \%$ of sections (horizontal black line) of area $X$ had an $0 \mathrm{D}$ ratio below the 95 th percentile of the sham distribution (gray region). $\boldsymbol{b}$, Red and blue symbols represent measurements taken from the left and right panels shown in $\boldsymbol{a}$, respectively.

reduced $\mathrm{TH}$-positive fiber density to $51 \%-84 \%$ of the normal value within the lesioned subregions of area X (Fig. 2e).

In the analyses shown in Figure $2 a-c$, we manually set an OD threshold to delineate the lesioned and nonlesioned subregions of area X (see Materials and Methods). To verify that these results were not an artifact of this procedure, we also performed an alternate analysis of lesion volume that did not rely on manual thresholding. As shown in Figure 3, this alternate analysis similarly found that 6-OHDA injections led to significant reductions in TH stain in approximately half of area X.

In addition to optical imaging of TH-stained sections, we also quantified the extent to which 6-OHDA injections reduced DA concentrations within area X using HPLC. In a separate cohort of Bengalese finches $(n=6)$, we injected one hemisphere with 6-OHDA and performed a sham lesion on the other hemisphere. This design allowed us to control for interindividual differences in neurotransmitter levels. As shown in Figure $4 a$, left, 6-OHDA lesions reduced the concentration of DA by an average of $47.1 \%$ (range 9.3\%-74.1\%, mean concentration 206.1 and $390.5 \mathrm{ng}$ $\mathrm{DA} / \mathrm{mg}$ protein in lesioned and sham hemispheres respectively).

6-OHDA is toxic to both dopaminergic and noradrenergic neurons, and $\mathrm{TH}$ staining labels both types of neurons. It was therefore important to consider the possibility that any observed effects of 6-OHDA injections on both behavior and $\mathrm{TH}$ stain density might reflect changes in noradrenergic input as well as (or instead of) changes in DA. However, we think that this possibility is extremely unlikely. Catecholaminergic innervation of area $\mathrm{X}$ has previously been shown to be overwhelmingly dopaminergic (Mello et al., 1998; Gale and Perkel, 2005; Castelino et al., 2007). Studies of noradrenergic inputs to area $\mathrm{X}$ have reported that such inputs are either absent (Mello et al., 1998) or extremely weak (Castelino et al., 2007), and NE concentration within area X has been reported to be $<3 \%$ of that of DA (Gale and Perkel, 2005). Consistent with these prior findings, NE concentrations assessed by HPLC were $<2 \%$ as great as DA concentrations in sham hemispheres (Fig. $4 a$, right; mean $1.2 \%$, range $0.5 \%-1.6 \%$, mean concentration 4.9 and $4.3 \mathrm{ng} \mathrm{NE} / \mathrm{mg}$ protein in lesioned and sham hemispheres, respectively), and furthermore were not significantly affected by 6-OHDA injections (Fig. 4b). Therefore, loss of noradrenergic inputs to area $\mathrm{X}$ is very unlikely to have affected our results.

Importantly, staining for the neuron-specific nuclear protein NeuN revealed that 6-OHDA injections did not reduce the number of neuronal somata within area $\mathrm{X}$ relative to sham injections (Fig. 5), suggesting that 6-OHDA injections reduced dopaminergic inputs without killing neuronal cell bodies in the basal ganglia. Additionally, we examined lesion-induced loss of dopaminergic neurons by counting TH-positive cell bodies in midbrain nuclei VTA/SNc and assessed lesion-related changes in noradrenergic neurons by counting TH-positive cells in the LC. Cell counts revealed no significant difference in either area $(2655 \pm 427$ mean \pm SD for VTA/SNc in sham birds; $2598 \pm 369$ for VTA/SNc in lesioned birds; $193 \pm 40$ for LC shams; $201 \pm 48$ for LC lesions; $p>0.8$ in all cases, Kolmogorov-Smirnov test). However, a post hoc power analysis (see Materials and Methods) revealed that we would be highly unlikely to detect the loss of $\mathrm{TH}$-positive neurons resulting from our injections of 6-OHDA given the fact that area $\mathrm{X}$ comprises only $\sim 10 \%$ of the total volume of the basal ganglia (Karten et al., 2013) and that our lesions affected only part of area X. Therefore, because of the very small volume of tissue injected with neurotoxin, the loss of TH-positive cell bodies in VTA/SNc known to follow 6-OHDA injections was well below our threshold for detectability.

Bilateral depletions of area X's dopaminergic innervation did not affect the amount or quality of song production. As shown in Figure $6 a, 6-O H D A$ injections had no significant effect on the number of songs produced per day, and the small changes in song number observed after neurotoxin injections were not significantly different from those that followed sham injections $(p>$ 0.05 , one- and two-sample Kolmogorov-Smirnov tests, respectively). Additionally, there were no detectable differences in the acoustic structure of song syllables. The gross spectral structure of song was unaffected by the lesions, as shown in example songs from the same bird before and after lesion (Fig. 6b). Quantitative acoustic analysis revealed that 6-OHDA injections caused no consistent changes in either the mean or variance of syllable pitch (Fig. $6 c$ ), sound amplitude, or spectral entropy $(p>0.25$, Kolmogorov-Smirnov tests).

Because increased DA within area X during female-directed song is associated with reductions in acoustic variability (Sasaki et al., 2006; Leblois et al., 2010; Leblois and Perkel, 2012; Murugan et al., 2013) depleting DA with 6-OHDA injections might cause vocal variability to increase in directed song even if it does not affect the variability of undirected song (Fig. 6c). Alternatively, because Parkinson's disease is associated with reductions in vocal variability (Ramig et al., 2008), 6-OHDA lesions might cause vocal variability to decrease. Accordingly, we collected pre- 
a

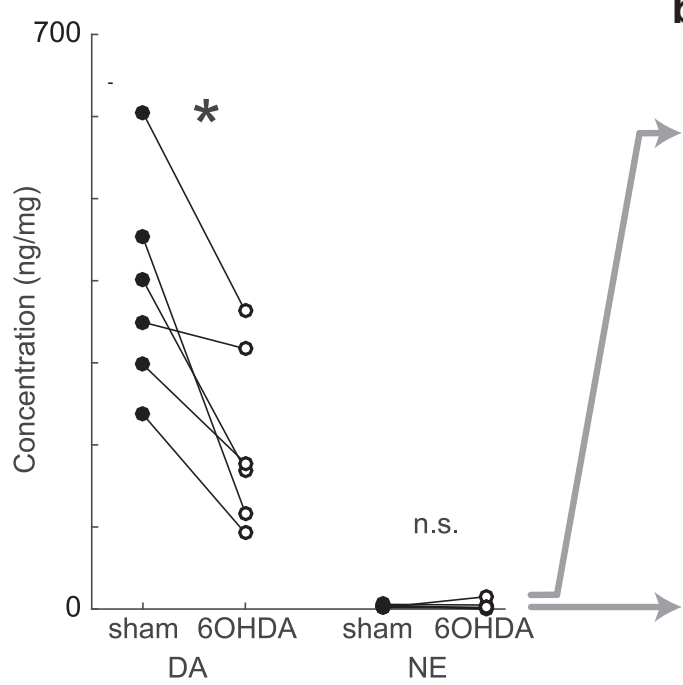

b

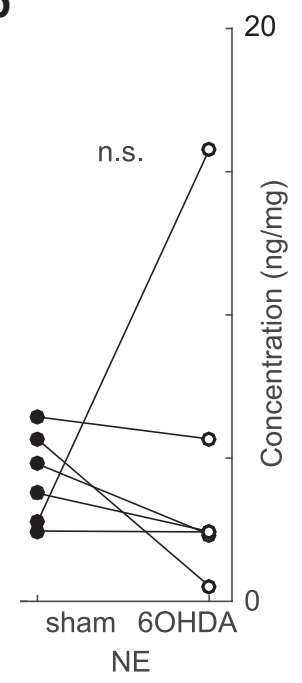

Figure 4. Concentrations of DA and NE in 6-OHDA- and sham-lesioned tissue. As described in Materials and Methods, we used HPLC to directly measure the concentration of DA and NE in birds that received 6-OHDA lesions of area X in one hemisphere and sham lesions in the other hemisphere. $\boldsymbol{a}$, In sham-lesioned hemispheres (filled symbols), the concentration of NE was extremely small relative to that of DA, with NE concentrations on average $1.2 \%$ as great as that of DA (range $0.5 \%-1.6 \%$ ). Injections of 6-OHDA significantly reduced the concentration of DA $\left({ }^{*} p<0.05\right.$, one-sample Kolmogorov-Smirnov test on normalized DA concentrations in the lesioned hemisphere) but did not significantly affect concentrations of NE. ( $p>0.8$; one-sample Kolmogorov-Smirnov test on normalized NE concentrations in the lesioned hemisphere). $\boldsymbol{b}$, Expanded view of NE data; note the difference in vertical scale between $\boldsymbol{a}$ and $\boldsymbol{b}$. n.s., Not significant.

lesion and postlesion directed song from 4 birds (see Materials and Methods) and analyzed pitch variability. Consistent with prior results (Kao et al., 2005; Kao and Brainard, 2006; Sakata et al., 2008), we found a lower pitch SD in prelesion directed versus undirected song $(p<0.05$, Wilcoxon signed-rank test; prelesion directed SD: mean 0.36 semitones, range $0.17-0.64$ semitones; prelesion undirected: $0.47,0.23-0.98)$. Interestingly, we did not find differences in either prelesion versus postlesion directed pitch SD or prelesion versus postlesion undirected pitch SD $(p>$ 0.05 , Wilcoxon signed-rank tests). Nor did we find a difference in postlesion directed versus undirected pitch SD $(p>0.05$, Wilcoxon signed-rank test). The $\sim 50 \%$ reduction in DA induced by 6-OHDA lesions (Figs. 2, 4) therefore appeared to have no significant effect on acoustic variability in either female-directed or undirected song.

However, we caution that these results are based on a relatively small dataset of directed song that is likely underpowered to detect subtle differences. Bengalese finches produce directed song much less readily than do zebra finches. Although we were able to collect some directed song (from 11 syllables prelesion and 8 syllables postlesion, with a mean of 110 and 88 iterations per syllable, respectively), attempting to collect more femaledirected song would have significantly impeded our examination of 6-OHDA's effect on learning because introducing female songbirds acutely reduces the total amount of song production. Importantly, we note that a recent study of the effects of 6-OHDA lesions of area $\mathrm{X}$ in zebra finches collected a much larger amount of female-directed song and reports significant variability changes after 6-OHDA lesions in area X (Miller et al., 2015).

In each bird, we compared vocal learning before and after either 6-OHDA or sham (saline) injections into area X (Fig. 1c). We evoked learning by providing disruptive auditory feedback (white noise blasts) conditional on the sung pitch of a particular syllable (Tumer and Brainard, 2007). In response to this reinforcement training, birds modify the pitch of the targeted syllable to avoid white noise, as shown in a representative prelesion experiment (Fig. $7 a$, black line, $b$ ). Following 6-OHDA injections, learning was greatly reduced in this bird (Fig. $7 a$, red line, c). Averaged across all subjects, the rate of learning decreased by $>50 \%$ following 6-OHDA injections (Fig. 7d), whereas no reduction was seen following sham surgeries (Fig. 7e).

In addition to directly comparing the time course of learning across the first $3 \mathrm{~d}$ of white noise (Fig. $7 a-f$ ), we also quantified learning on trial-matched prelesion and postlesion days (see Materials and Methods) and similarly found reduced learning in 6-OHDA-lesioned (Fig. 7g) but not sham-lesioned birds (Fig. 7h). This analysis addresses a confound that could arise if we interpreted learning solely on a chronological basis (Fig. $7 a-f$ ). Because of normal variation in singing rate, birds sang a somewhat different number of songs by postlesion day 3 than by prelesion day 3 . Hence, if a particular bird learned less by postlesion day 3 (compared with prelesion day 3 ), it could occur because that bird experienced substantially fewer learning trials, not because of DA lesion. Likewise, if a bird learned the same amount by postlesion day 3 , it could occur because that bird experienced substantially more trials, masking a DA-dependent learning deficit. Therefore, it is crucial to compare prelearning/postlearning both chronologically (Fig. 7a-f) and using a postlesion day where birds had a similar number of trials (within 10\%) as in the prelesion experiment (Fig. $7 g-i$ ). Additionally, because difference between $p$ values does not always correspond to a difference between effects (Nieuwenhuis et al., 2011), we directly compared the (post - pre) learning changes between conditions and found significantly reduced learning in 6-OHDA birds (Fig. 7i). To our knowledge, these results provide the first direct evidence that vocal learning in songbirds depends strongly on dopaminergic input to the basal ganglia.

As described in Materials and Methods, animals were randomly assigned to either the 6-OHDA lesion or sham group. Notably, two of the birds randomly assigned to the lesion group exhibited stronger prelesion learning than did their counterparts in the presham group (compare black symbols showing "prelesion" and "pre-sham" learning values in Fig. $7 g$ and 7 Fig. $7 h$, respectively). As a result, the prelesion learning data combined across subjects (Fig. $7 d$, black trace) exhibited noticeably greater learning than the presham data (Fig. $7 e$, black trace). To assess whether the apparent effects of 6-OHDA on learning could have arisen from a difference in prelesion/presham learning ability, we repeated the analysis in Figure $7 d$ after excluding the two animals that exhibited the greatest prelesion learning. As shown in Figure $7 f$, this reanalysis yields comparable prelesion/presham learning (compare black traces in Fig. $7 e$ and $7 f$ ) and, similar to the full dataset from lesioned animals, reveals a significant drop in learning ability following 6-OHDA injection, demonstrating that the 6-OHDA-dependent reduction in learning shown in Figure $7 d, e$ was not an artifact of a difference in learning ability in the two subject groups before lesion or sham injections. 
We used a stepwise regression procedure to quantify whether the size or location of the loss of dopaminergic input within area $\mathrm{X}$ predicted the magnitude of behavioral effects shown in Figure $7 g$. We found that none of the candidate predictor values, which included the total fraction of area $\mathrm{X}$ in which $\mathrm{TH}$ stain was reduced $\left(\alpha_{\text {Total }}\right)$ as well as the fraction within six different subregions of area $\mathrm{X}$ (anterior, posterior, medial, lateral, dorsal and ventral), were significantly correlated with either the absolute or relative change in vocal plasticity $\left(\Delta_{\text {Absolute }}\right.$ or $\Delta_{\text {Relative; }}$; see Materials and Methods), either as individual predictors or in any combination. However, it should be noted that our dataset contains a somewhat limited range of lesion sizes (Fig. 2c), potentially limiting our ability to identify such effects. Notably, anatomical studies have shown a topographic mapping between different subregions of area $\mathrm{X}$ and downstream components of the song system (Luo et al., 2001), suggesting that different portions of area $\mathrm{X}$ might be dedicated to the modification of particular vocal parameters, such as pitch (Luo et al., 2001; Fee and Goldberg, 2011). Although our analysis did not produce positive evidence for such specificity, the spread of 6-OHDA within area $\mathrm{X}$ prevented us from fully assessing this idea by precisely confining lesions to particular subregions of the nucleus.

Following training, we turned off white noise playbacks and continued to monitor vocal acoustics for at least $3 \mathrm{~d}$ (see Materials and Methods). In contrast to the large deficits in learning observed during operant conditioning (Fig. 7), 6-OHDA lesions did not appear to impair spontaneous pitch restoration after learning. Both before and after lesion, the pitch of song changed monotonically toward the baseline (pretraining) value (Fig. $8 a$ ). Furthermore, quantifying the time constant of restoration demonstrated that pitch actually recovered significantly faster after 6-OHDA lesion than prelesion $\left(\tau_{\text {prelesion }}=2.15 \mathrm{~d}, \tau_{\text {postlesion }}=0.87 \mathrm{~d}\right.$; Fig. $8 b)$. However, the faster time constant of restoration postlesion does not necessarily reflect enhanced learning after 6-OHDA lesions. Rather, the observed difference in time constant may reflect the well-established phenomenon (Sober and Brainard, 2012; Kelly and Sober, 2014) that learning speed increases when the experienced sensory error is smaller relative to baseline. Indeed, in postlesion experiments, birds began restoration with a smaller error because learning was impaired (compare the last white noise day for prelesion and postlesion experiments in Fig. $8 a$ ). The fit parameter $p_{\text {final }}$, which estimates the eventual equilibrium state of learning, was close to zero in both cases ( $p_{\text {final }}=-0.04$ semitones prelesion,

b Not significant.
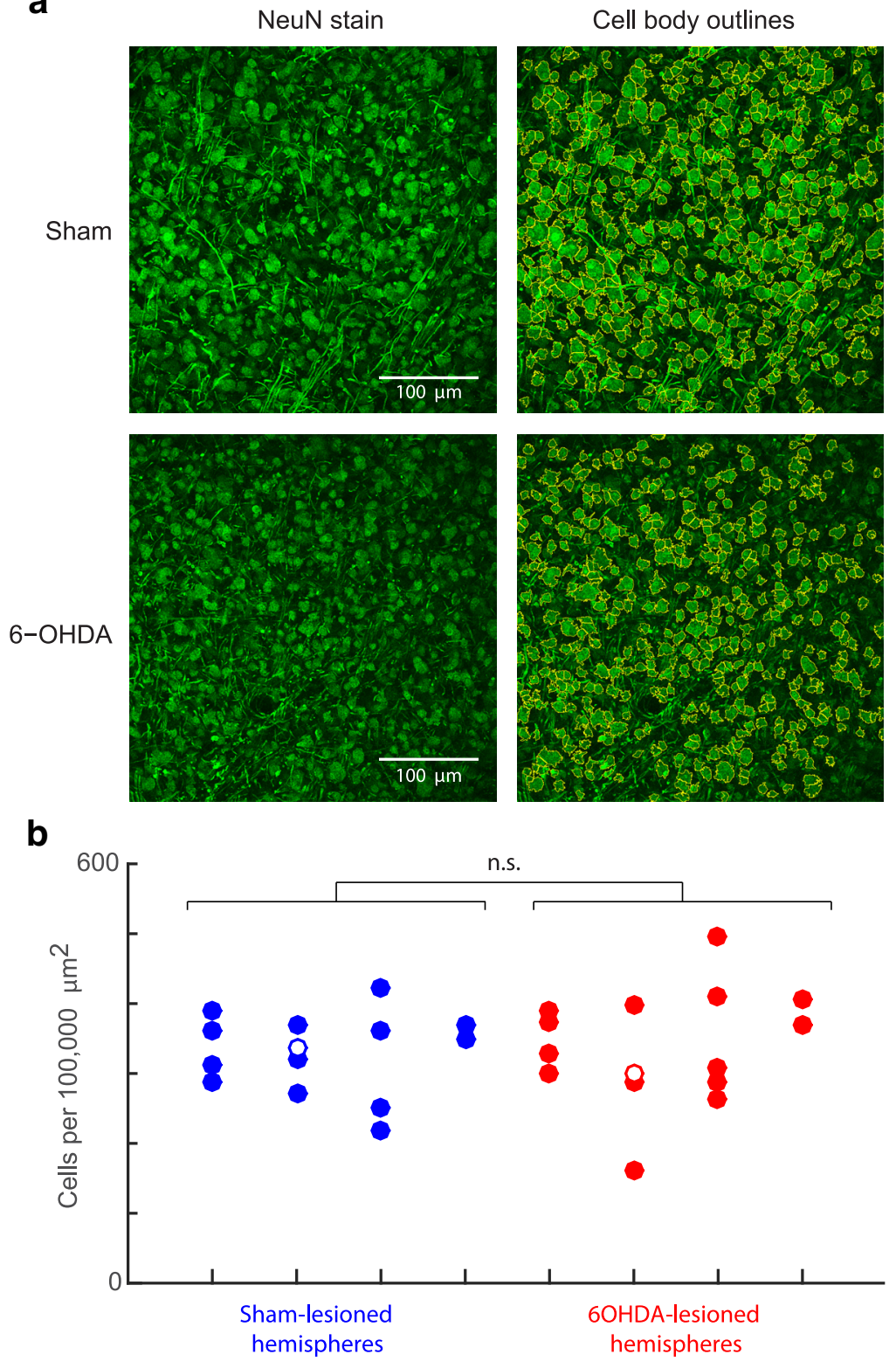

Figure 5. 6-OHDA injections do not lead to neuron loss within area $X . a$, Representative NeuN-stained images from birds that received sham (top) and 6-OHDA (bottom) lesions. In each section, we counted the number of neuronal cell bodies (right column; see Materials and Methods). $\boldsymbol{b}$, Area Ximages were taken from two bilaterally and two unilaterally lesioned birds (each $369 \times 369$ $\mu \mathrm{m})$. Blue and red circles represent the number of cell bodies in individual sections. Open circles represent the values for the example sham and 6-OHDA lesions shown in $\boldsymbol{a}$. All images from 6-OHDA-injected hemispheres were taken from within subregions of area $X$ that exhibited significant loss of TH staining. We did not detect a significant difference in the number of cell bodies in area $X$ in 6-OHDA versus sham conditions ( $p>0.7$, partial $F$ test; see Materials and Methods). This suggests that, while lesions decreased dopaminergic inputs to area $X$ (Figs. 2, 3, 4), 6-0HDA injections did not kill neurons with cell bodies within area X. n.s.,

$p_{\text {final }}=0.05$ semitones postlesion), suggesting that both prelesion and postlesion animals would have returned pitch to near the baseline value had washout been allowed to run for longer than the three post-white noise washout days shown in Figure $8 a$.

To control for the fact that learning speed depends on sensory error magnitude, we further examined the effects of DA lesion on pitch restoration by comparing postlesion restoration (Fig. 8, red traces) with data from specially selected subsets of experiments performed in the non-6-OHDA-lesioned condition. Specifically, we created these subsampled datasets by progressively eliminating the nonlesioned animals with the greatest learning on the last 

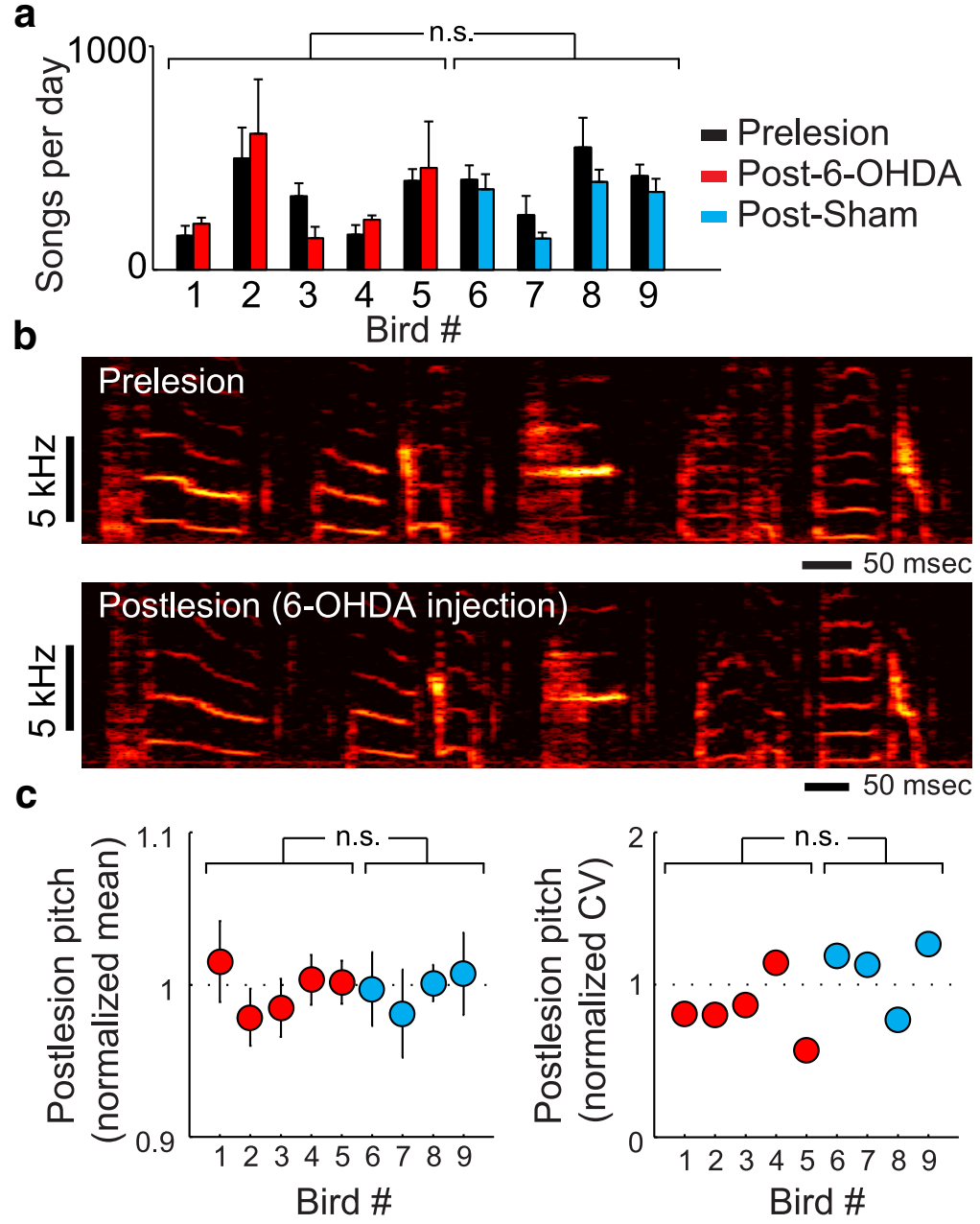

Figure 6. Removal of DA inputs to area X does not degrade song quantity or quality. $\boldsymbol{a}$, The number of song bouts produced per day did not significantly differ in 6-0HDA-injected versus sham-lesioned animals (see Results). $\boldsymbol{b}$, Spectrograms represent the acoustic power (color scale) at different frequencies as a function of time for two representative samples of a bird's song before (top) and $5 \mathrm{~d}$ after (bottom) bilateral 6-OHDA injections into area X. c, Across animals, there were no consistent differences in the mean (left) or variability (CV; right) of the pitch of the song syllables targeted with white noise when the postlesion data were normalized by their prelesion values ( $p>0.5$, Kolmogorov-Smirnov tests) in birds receiving 6-0HDA injections (red) or sham lesions (blue). n.s., Not significant.

white noise day until the remaining nonlesioned animals showed nearly identical vocal errors on the final white noise day as did the DA-lesioned population. Figure $8 c$ shows a version of this analysis in which we compared all post-6-OHDA animals (red, $n=5$ experiments) with data selected from prelesion, presham, and postsham animals so that the selected nonlesioned dataset ("Non-6-OHDA (selected)," $n=6$ experiments; Fig. $8 c$, blue trace) had approximately the same initial error as the postlesion data (Fig. $8 c$, red trace). In an alternate version of this analysis (Fig. 8e), we selected the nonlesioned datasets only from postsham animals ("Postsham (selected)," $n=2$ experiments). In both cases, these alternate analyses (Fig. $8 d, f$ ) yielded qualitatively the same results as those shown in the initial analysis (Fig. $8 b$ ), with significantly faster learning after 6-OHDA lesion (Fig. $8 d, f$, asterisks) and $p_{\text {final }}$ values very close to zero $\left(p_{\text {final }}=0.004\right.$ and 0.1 semitones for the nonlesioned data shown in Fig. $8 d$ and Fig. $8 f$, respectively). Thus, although analysis of our relatively short washout period does not allow us to make strong conclusions regarding the effects of DA lesions on spontaneous error correction, our analyses clearly indicate that restoration back to pitch baseline is not impaired by reduction of dopaminergic in-
- $50 \mathrm{msec}$

puts to area $\mathrm{X}$, as is learning guided by white noise, and indeed may be facilitated by 6-OHDA lesions (see Discussion).

\section{Discussion}

Our experiments show that 6-OHDA injections into the songbird basal ganglia nucleus area $\mathrm{X}$ caused significant loss of DA inputs without causing detectable loss of neurons within area X. These dopaminergic lesions caused significant vocal learning deficits when pitch changes were driven by white noise reinforcement but did not result in measurable changes in song performance, song variability, or pitch restoration to baseline after white noise was discontinued. These results suggest dopaminergic inputs to the basal ganglia are critical for guiding vocal learning, at least when learning is driven by external reinforcement signals.

Although we took pains to precisely target 6-OHDA injections to area $\mathrm{X}$, and indeed loss of $\mathrm{TH}$ stain was mostly confined to this nucleus, in some cases we observed loss of label in the striatum immediately surrounding area X (Fig. 3a). Importantly, the "shell" region immediately surrounding area $\mathrm{X}$ is hypothesized to be part of a circuit parallel to the classical song system shown in Figure $1 a$. Although cortical components of this parallel system appear to contribute to vocal learning (Iyengar et al., 1999; Person et al., 2008; Bottjer and Altenau, 2010), the involvement of area $\mathrm{X}_{\text {shell }}$ in learning remains to be directly tested. Because some spillover of 6-OHDA is inevitable, we cannot exclude the possibility that some of the observed effects on vocal learning reflect loss of dopaminergic input to the shell surrounding area X. However, we consider this possibility unlikely given that the loss of label outside of area $\mathrm{X}$ affected a very small fraction of the surrounding striatum.

Because female-directed song has lower acoustic variability and is associated with increased DA in area X (Sasaki et al., 2006; Leblois et al., 2010; Leblois and Perkel, 2012; Murugan et al., 2013), we expected to see increased vocal variability after 6-OHDA lesions. However, we did not observe a significant change in pitch variability in either direction following 6-OHDA injections for undirected (Fig. $6 c$ ) or directed song, consistent with another study finding no changes in syllable structure after unilateral 6-OHDA lesions in VTA/SNc (Hara et al., 2007). The lack of an effect of lesions on social context-dependent variability may reflect the incomplete nature of our lesions, which spared a substantial number of DA terminals within area X. Alternatively, it is possible that neuromodulatory factors in addition to DA or neural circuits other than area $\mathrm{X}$ also contribute to contextdependent changes in variability and were able to compensate for lesion-induced changes.

Notably, a recent study analyzed vocal variability after 6-OHDA lesions of dopaminergic input to area $\mathrm{X}$ in zebra finches 
a

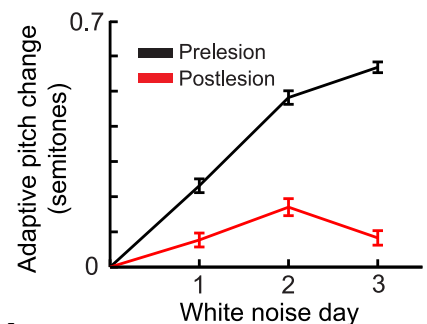

d

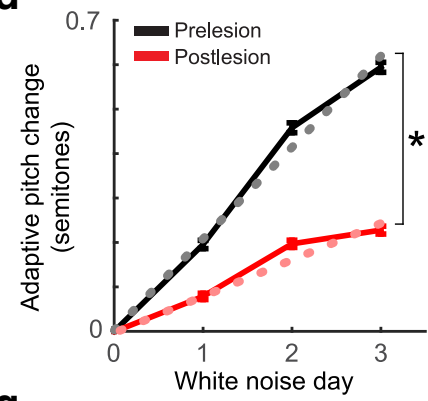

g

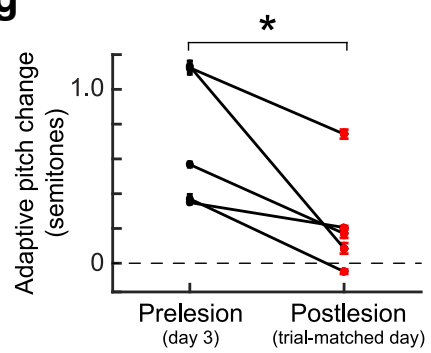

b

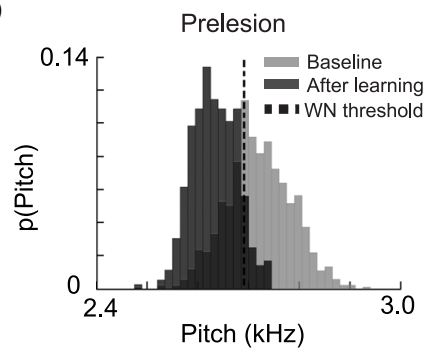

e

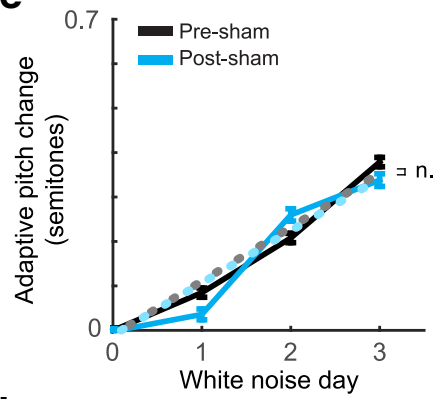

h

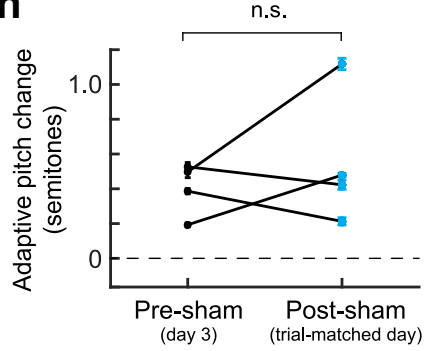

C

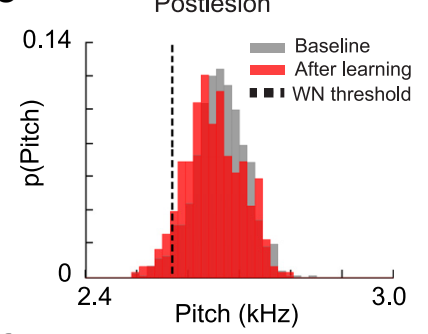

f

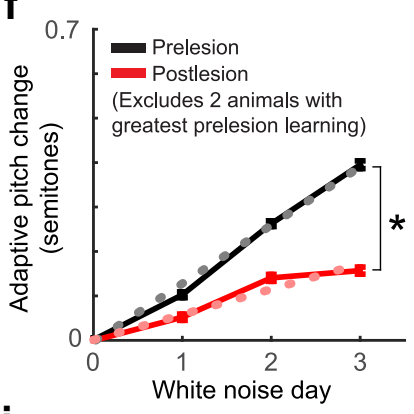

i

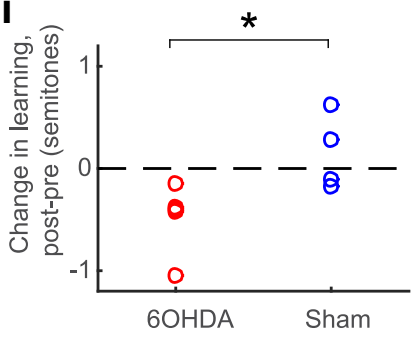

Figure 7. Removal of DA inputs to area X impairs reinforcement-driven vocal learning. $\boldsymbol{a}$, In an example experiment, a bird received $3 \mathrm{~d}$ of training in which higher-pitched renditions of a syllable were punished by a disruptive auditory stimulus (see Materials and Methods). Black and red traces represent the pitch of the targeted syllable (mean \pm SEM) before and after 6-OHDA injections, respectively, and illustrate a substantial reduction in learning magnitude following lesion. Pitch changes in the adaptive direction (downwards) are plotted as positive values. $\boldsymbol{b}, \boldsymbol{c}$, Prelesion (b) and postlesion (c) pitch distributions for the experiment shown in $\boldsymbol{a}$. Gray bars represent the $3 \mathrm{~d}$ baseline pitch distribution. Dashed lines indicate the threshold for white noise playback (i.e., any pitches sung above that threshold received white noise). In every experiment, learning was driven in the same syllable and in the same direction prelesion and postlesion. $\boldsymbol{d}$, Group data for lesioned (6-OHDA-injected) animals. Solid lines indicate the pitch of the targeted syllable as in $\boldsymbol{a}$, except that here data are combined across $n=5$ lesioned animals. Dotted lines indicate linear regression to pitch data. ${ }^{*} p<0.0001$, significantly different slopes ( $F$ test). $\boldsymbol{e}$, Group data for $n=4$ sham-lesioned animals, plotting and testing conventions as in $\boldsymbol{d}$. Slopes of pitch as a function of time are not significantly different ( $p=0.48, F$ test). $\boldsymbol{f}$, Alternate analysis of data from 6-0HDA-lesioned animals, excluding the two subjects who showed the greatest prelesion learning (see Results and $\boldsymbol{g}$ ). Plotting and testing conventions as in $\boldsymbol{d}$; pitch slopes are significantly different $\left({ }^{*} p<0.0001, F\right.$ test). $\boldsymbol{g}$, Adaptive pitch change on the last white noise day in the prelesion experiment (relative to baseline) versus adaptive pitch change on a trial-matched white noise day in the postlesion experiment (not necessarily day 3 ; see Materials and Methods). ${ }^{*} p<0.05$, significant difference in prelesion and postlesion learning magnitude (one-sided Wilcoxon signed-rank test). $\boldsymbol{h}$, Adaptive pitch change on the last white noise day for sham-lesioned animals (conventions as in $\boldsymbol{g}$ ). $\boldsymbol{i}$, Direct comparison between sham and 6-0HDA learning changes. ${ }^{*} p<0.05$ (two-sample $t$ test). n.S., Not significant.

(Miller et al., 2015). Contrary to our hypothesis, this study found significant reductions in undirected (but not female-directed) song variability, similar to the decreases in vocal variability observed in Parkinson's disease (Ramig et al., 2008). Our conflicting results may be attributed to two factors beyond the obvious difference in the species being studied. First, as noted in Results, we had a relatively small sample size of interleaved directed/undirected songs, lowering our statistical power to detect subtle differences in variability. Second, our average 6-OHDA dosage was slightly higher (1.3 $\mu \mathrm{g}$; Table 1$)$, and we quantified variability at later time periods (13-22 d after lesion for directed song analysis compared with 4-5 d after lesion in Miller et al., 2015), raising the possibility of a complex relationship between the extent of DA depletion, time course of compensation, and changes in vocal variability. The effects of DA depletion on vocal variability in Bengalese finches therefore remain to be clarified by future studies.

Although 6-OHDA lesions caused vocal learning deficits during white noise training (Fig. 7), restoration to baseline pitch was not impaired during the washout phase (Fig. 8), suggesting that DA might play different roles in distinct forms of vocal learning. Wholesale lesions or inactivation of area X or LMAN (the output nucleus of the AFP) severely degrade both white noise-driven pitch learning and spontaneous restoration back to baseline (Andalman and Fee, 2009; Warren et al., 2011; Charlesworth et al., 2012; Ali et al., 2013). Our data indicating that only the former process depends on dopaminergic inputs to area $\mathrm{X}$ suggest that the basal ganglia might mediate these two forms of vocal plasticity in distinct ways. Interestingly, lesions in caudal medial nidopallium, proposed as a candidate site for template song memory, disrupt restoration but spare noise-avoidance learning (Canopoli et al., 2014), the opposite pattern we observed following dopaminergic lesions to area $\mathrm{X}$. These observations support the idea that "vocal plasticity" can be divided into different subtypes, each driven by distinct yet interacting neural processes.

Despite the above considerations, however, our data suggesting that restoration is less impeded by DA loss than is noisedriven learning should be treated with a great deal of caution. 
a
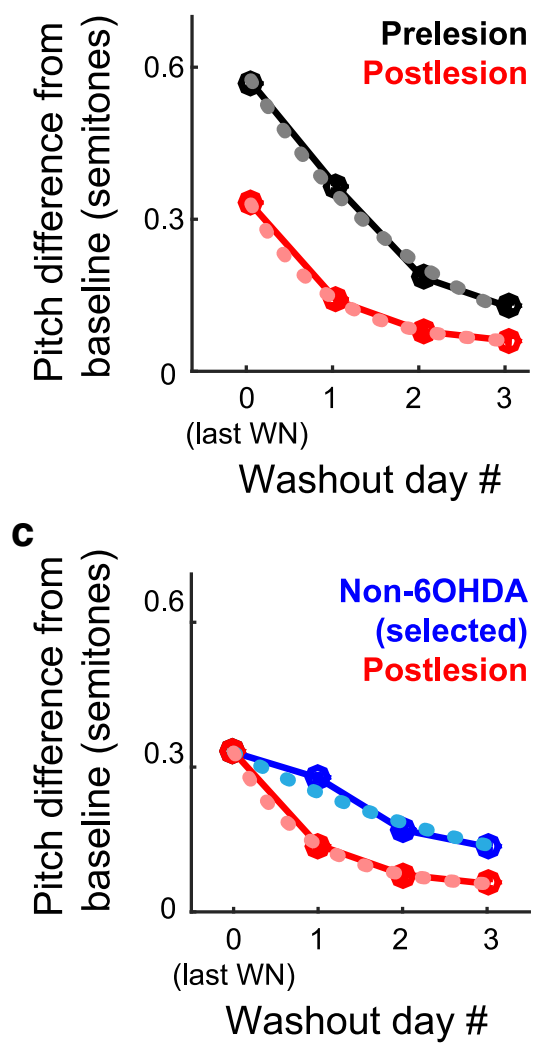

e

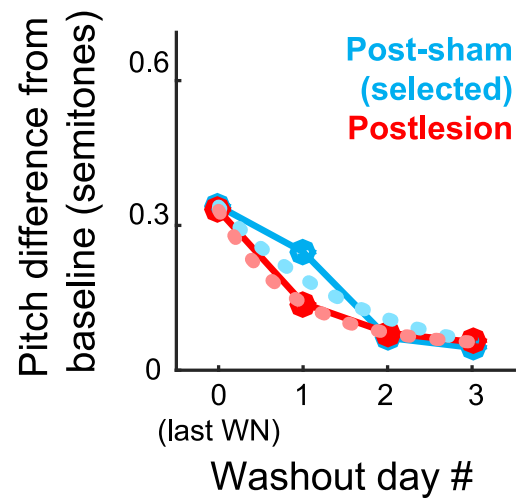

b

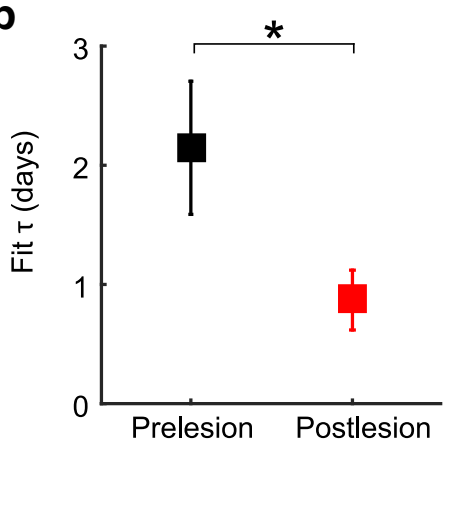

d

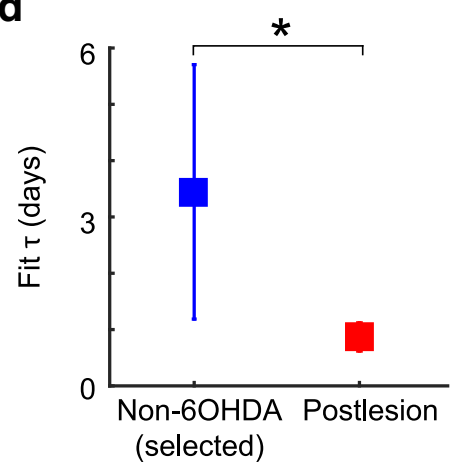

f

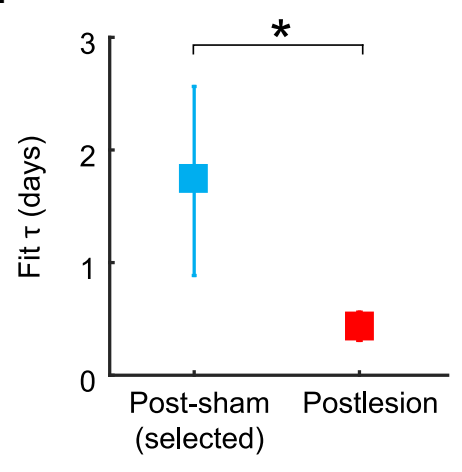

Figure 8. Removal of DA inputs to area X does not impair pitch restoration. $\boldsymbol{a}$, Combined data across five 6-0HDA-lesioned birds during the restoration period, after white noise was discontinued. Washout day 0 is the last day of white noise (not necessarily day 3; see Materials and Methods). Black and red represent prelesion and postlesion experiments, respectively. Prelesion and postlesion restoration data were fit with an exponential decay model (dashed lines; see Materials and Methods). Birds restored pitch toward baseline in both prelesion and postlesion experiments. After 6-0HDA lesions, birds began with a lower absolute pitch difference from baseline because of postlesion learning deficits (Fig. 7d). $\boldsymbol{b}$, Fitted time constant $\tau$ for prelesion and postlesion learning. Lower $\tau$ indicates faster return to baseline. In postlesion experiments, birds restored pitch significantly faster than in prelesion experiments ( ${ }^{*} p<0.05$, permutation test). Error bars indicate $95 \%$ confidence intervals. c, d, Same analysis as in $\boldsymbol{a}, \boldsymbol{b}$, but selecting nonlesioned datasets so as to approximately equalize the initial error (i.e., to approximately equalize error on the last white noise day). Here nonlesioned datasets are selected from prelesion, presham, and postsham subjects (see Materials and Methods). $\boldsymbol{e}, \boldsymbol{f}$, Same analysis as in $\boldsymbol{c}, \boldsymbol{d}$, but with nonlesioned datasets drawn only from postsham subjects. Note different vertical scale in $\boldsymbol{d}$ compared with that in $\boldsymbol{b}, \boldsymbol{f} . \boldsymbol{a}, \boldsymbol{c}, \boldsymbol{e}$, SEM error bars are obscured by the plotted circles. *

First, our 6-OHDA injections only partially ablated DA within area $\mathrm{X}$, and the robust restoration observed after lesion might reflect residual DA function. Second, because washout experiments necessarily occurred after white noise training, it is possible that some form of compensatory plasticity occurred in the few days that elapsed between the onset of postlesion white noise training and the beginning of the washout period. Third, it is important to emphasize that the return to baseline is not necessarily auditory-guided and could in theory be mediated by a bird's matching proprioceptive feedback to the baseline motor command. Finally, as shown in Figure $8 a, c, e$, we did not collect washout data for sufficient time for syllable acoustic to fully return to baseline either prelesion or postlesion. Therefore, although the speed of restoration appears unimpeded by 6-OHDA lesions, the endpoint of restoration following DA depletion remains to be measured directly. Intriguingly, the analyses shown in Figure 8 suggest that pitch restoration may actually progress more quickly following DA lesions, even when selecting subsets of the data so as to equalize the initial error size (Fig. $8 c-f$ ). Although the caveats detailed above prevent us from drawing strong conclusions about DA's role in vocal learning other than that driven by white noise, future studies could ask whether DA is necessary for error-corrective learning by providing a correctable auditory perturbation to baseline song without explicit external reinforcement (Sober and Brainard, 2009; Hoffmann et al., 2012).

Prior studies have identified potential mechanisms by which dopaminergic inputs to area $\mathrm{X}$ might mediate vocal learning. The nuclei of dopaminergic neurons that project to area X reside in the VTA/ SNc complex, which in turn receives input from forebrain neurons that respond to perturbations of auditory feedback during singing (Mandelblat-Cerf et al., 2014), providing a candidate pathway by which sensory error signals might influence DA release within the basal ganglia. Furthermore, dopaminergic signaling plays a crucial role in mediating plasticity at corticostriatal synapses in both mammals and songbirds (Ding and Perkel, 2004; Surmeier et al., 2007; Leblois, 2013), suggesting that DA might mediate vocal learning by modulating changes in synaptic strength between cortical area HVC and spiny neurons in area X (Fee and Goldberg, 2011). Although our results strongly suggest that dopaminergic projections from VTA/SNc guide vocal plasticity, future studies (including recording from DA neurons that project to area $\mathrm{X}$ ) are needed to investigate the nature of the signals conveyed by these projections.

Our lesions only partially eliminated dopaminergic inputs to area $\mathrm{X}$, in contrast to studies in mammals in which injections of neurotoxins into the medial forebrain bundle produces nearcomplete ipsilateral loss of dopaminergic input throughout the striatum (Deumens et al., 2002). Specifically, our lesions reduced 
TH stain in approximately half of area X (Fig. 2c), and within the affected regions eliminated $\sim 15 \%-50 \%$ of catecholaminergic axons (Fig. 2e), comparable with the fiber loss observed following intrastriatal 6-OHDA injections in mammals (Debeir et al., 2005). HPLC results similarly showed DA concentration dropping $47 \%$ on average (Fig. $4 a$ ). Because even these relatively modest reductions in dopaminergic innervation produced learning deficits, our results demonstrate that vocal learning is sensitive to disruptions of dopaminergic input to the basal ganglia and suggest that DA plays a crucial role in the processing of sensorimotor errors.

\section{References}

Ali F, Otchy TM, Pehlevan C, Fantana AL, Burak Y, Ölveczky BP (2013) The basal ganglia is necessary for learning spectral, but not temporal, features of birdsong. Neuron 80:1-13. CrossRef Medline

Andalman AS, Fee MS (2009) A basal ganglia-forebrain circuit in the songbird biases motor output to avoid vocal errors. Proc Natl Acad Sci U S A 106:12518-12523. CrossRef Medline

Beeler JA, Cao ZF, Kheirbek MA, Ding Y, Koranda J, Murakami M, Kang UJ, Zhuang X (2010) Dopamine-dependent motor learning insight into Levodopa's long-duration response. Ann Neurol 67:639-647. CrossRef Medline

Bottjer SW, Altenau B (2010) Parallel pathways for vocal learning in basal ganglia of songbirds. Nat Neurosci 13:153-155. CrossRef Medline

Brainard MS, Doupe AJ (2000) Interruption of a basal ganglia-forebrain circuit prevents plasticity of learned vocalizations. Nature 404:762-766. CrossRef Medline

Canopoli A, Herbst JA, Hahnloser RH (2014) A higher sensory brain region is involved in reversing reinforcement-induced vocal changes in a songbird. J Neurosci 34:7018-7026. CrossRef Medline

Castelino C, Diekamp B, Ball G (2007) Noradrenergic projections to the song control nucleus area $\mathrm{X}$ of the medial striatum in male zebra finches (Taeniopygia guttata). J Comp Neurol 562:544-562. CrossRef Medline

Charlesworth JD, Warren TL, Brainard MS (2012) Covert skill learning in a cortical-basal ganglia circuit. Nature 486:251-255. CrossRef Medline

Colombo M (2014) Deep and beautiful: the reward prediction error hypothesis of dopamine. Stud Hist Philos Biol Biomed Sci 45:57-67. CrossRef Medline

Debeir T, Ginestet L, François C, Laurens S, Martel JC, Chopin P, Marien M, Colpaert F, Raisman-Vozari R (2005) Effect of intrastriatal 6-OHDA lesion on dopaminergic innervation of the rat cortex and globus pallidus. Exp Neurol 193:444-454. CrossRef Medline

Deumens R, Blokland A, Prickaerts J (2002) Modeling Parkinson's disease in rats: an evaluation of 6-OHDA lesions of the nigrostriatal pathway. Exp Neurol 175:303-317. CrossRef Medline

Ding L, Perkel DJ (2004) Long-term potentiation in an avian basal ganglia nucleus essential for vocal learning. J Neurosci 24:488-494. CrossRef Medline

Doya K, Sejnowski T (1998) A computational model of birdsong learning by auditory experience and auditory feedback. In: Central auditory processing and neural modeling, pp 77-88. New York: Springer.

Draper NR, Smith H (1998) Applied regression analysis, Ed 3. New York: Wiley.

Eckart MT, Huelse-Matia MC, McDonald RS, Schwarting RK (2010) 6-Hydroxydopamine lesions in the rat neostriatum impair sequential learning in a serial reaction time task. Neurotox Res 17:287-298. CrossRef Medline

Fee MS, Goldberg JH (2011) A hypothesis for basal ganglia-dependent reinforcement learning in the songbird. Neuroscience 198:152-170. CrossRef Medline

Gale SD, Perkel DJ (2005) Properties of dopamine release and uptake in the songbird basal ganglia. J Neurophysiol 93:1871-1879. CrossRef Medline

Graybiel AM (2005) The basal ganglia: learning new tricks and loving it. Curr Opin Neurobiol 15:638-644. CrossRef Medline

Hara E, Kubikova L, Hessler NA, Jarvis ED (2007) Role of the midbrain dopaminergic system in modulation of vocal brain activation by social context. Eur J Neurosci 25:3406-3416. CrossRef Medline

Hoffmann LA, Kelly CW, Nicholson DA, Sober SJ (2012) A lightweight, headphones-based system for manipulating auditory feedback in songbirds. J Vis Exp 69:e50027. CrossRef Medline
Iyengar S, Viswanathan SS, Bottjer SW (1999) Development of topography within song control circuitry of zebra finches during the sensitive period for song learning. J Neurosci 19:6037-6057. Medline

Kao MH, Brainard MS (2006) Lesions of an avian basal ganglia circuit prevent context-dependent changes to song variability. J Neurophysiol 96: 1441-1455. CrossRef Medline

Kao MH, Doupe AJ, Brainard MS (2005) Contributions of an avian basal ganglia-forebrain circuit to real-time modulation of song. Nature 433: 638-643. CrossRef Medline

Karten HJ, Brzozowska-Prechtl A, Lovell PV, Tang DD, Mello CV, Wang H, Mitra PP (2013) Digital atlas of the zebra finch (Taeniopygia guttata) brain: a high-resolution photo atlas. J Comp Neurol 521:3702-3715. CrossRef Medline

Kelly CW, Sober SJ (2014) A simple computational principle predicts vocal adaptation dynamics across age and error size. Front Integr Neurosci 8:75. CrossRef Medline

Kutner MH, Nachtsheim CJ, Neter J, Li W (2005) Applied linear statistical models. New York: McGraw Hill.

Lang AE, Obeso JA (2004) Time to move beyond nigrostriatal dopamine deficiency in Parkinson's disease. Ann Neurol 55:761-765. CrossRef Medline

Leblois A (2013) Social modulation of learned behavior by dopamine in the basal ganglia: insights from songbirds. J Physiol 107:219-229. CrossRef Medline

Leblois A, Perkel DJ (2012) Striatal dopamine modulates song spectral but not temporal features through D1 receptors. Eur J Neurosci 35:17711781. CrossRef Medline

Leblois A, Wendel BJ, Perkel DJ (2010) Striatal dopamine modulates basal ganglia output and regulates social context-dependent behavioral variability through D1 receptors. J Neurosci 30:5730-5743. CrossRef Medline

Lowry OH, Rosebrough NJ, Farr LA, Randall RJ (1951) Protein measurement with the folin phenol reagent. J Biol Chem 193:265-275. Medline

Luo M, Ding L, Perkel DJ (2001) An avian basal ganglia pathway essential for vocal learning forms a closed topographic loop. J Neurosci 21:68366845. Medline

Mandelblat-CerfY, Las L, Denisenko N, Fee M (2014) A role for descending auditory cortical projections in songbird vocal learning. eLife 16:3. CrossRef Medline

Mello CV, Pinaud R, Ribeiro S (1998) Noradrenergic system of the zebra finch brain: immunocytochemical study of dopamine- $\beta$-hydroxylase. J Comp Neurol 400:207-228. CrossRef Medline

Miller JE, Hafzalla GW, Burkett ZD, Fox CM, White SA (2015) Reduced vocal variability in a zebra finch model of dopamine depletion: implications for Parkinson disease. Physiol Rep 3:e12599. CrossRef Medline

Mollaei F, Shiller DM, Gracco VL (2013) Sensorimotor adaptation of speech in Parkinson's disease. Mov Disord 28:1668-1674. CrossRef Medline

Murugan M, Harward S, Scharff C, Mooney R (2013) Diminished FoxP2 levels affect dopaminergic modulation of corticostriatal signaling important to song variability. Neuron 80:1464-1476. CrossRef Medline

Nieuwenhuis S, Forstmann BU, Wagenmakers EJ (2011) Erroneous analyses of interactions in neuroscience: a problem of significance. Nat Neurosci 14:1105-1107. CrossRef Medline

Ogura T, Ogata M, Akita H, Jitsuki S, Akiba L, Noda K, Hoka S, Saji M (2005) Impaired acquisition of skilled behavior in rotarod task by moderate depletion of striatal dopamine in a pre-symptomatic stage model of Parkinson's disease. Neurosci Res 51:299-308. CrossRef Medline

Palkovits M (1973) Isolated removal of hypothalamic or other brain nuclei of the rat. Brain Res 59:449-450. CrossRef Medline

Peh WY, Roberts TF, Mooney R (2015) Imaging auditory representations of song and syllables in populations of sensorimotor neurons essential to vocal communication. J Neurosci 35:5589-5605. CrossRef Medline

Person AL, Gale SD, Farries MA, Perkel DJ (2008) Organization of the songbird basal ganglia, including area X. J Comp Neurol 508:840-866. CrossRef Medline

Pozdeyev N, Tosini G, Li L, Ali F, Rozov S, Lee RH, Iuvone PM (2008) Dopamine modulates diurnal and circadian rhythms of protein phosphorylation in photoreceptor cells of mouse retina. Eur J Neurosci 27: 2691-2700. CrossRef Medline

Preibisch S, Saalfeld S, Tomancak P (2009) Globally optimal stitching of tiled 3D microscopic image acquisitions. Bioinformatics 25:1463-1465. CrossRef Medline 
Ramig LO, Fox C, Sapir S (2008) Speech treatment for Parkinson's disease. Expert Rev Neurother 8:297-309. CrossRef Medline

Sakata JT, Hampton CM, Brainard MS (2008) Social modulation of sequence and syllable variability in adult birdsong. J Neurophysiol 99: 1700-1711. CrossRef Medline

Sasaki A, Sotnikova TD, Gainetdinov RR, Jarvis ED (2006) Social contextdependent singing-regulated dopamine. J Neurosci 26:9010-9014. CrossRef Medline

Scharff C, Nottebohm F (1991) A comparative study of the behavioral deficits following lesions of various parts of the zebra finch song system: implications for vocal learning. J Neurosci 11:2896-2913. Medline

Schober A (2004) Classic toxin-induced animal models of Parkinson's disease: 6-OHDA and MPTP. Cell Tissue Res 318:215-224. CrossRef Medline

Schultz W (2007) Behavioral dopamine signals. Trends Neurosci 30:203210. CrossRef Medline

Schultz W (2013) Updating dopamine reward signals. Curr Opin Neurobiol 23:229-238. CrossRef Medline

Sober SJ, Brainard MS (2009) Adult birdsong is actively maintained by error correction. Nat Neurosci 12:927-931. CrossRef Medline

Sober SJ, Brainard MS (2012) Vocal learning is constrained by the statistics of sensorimotor experience. Proc Natl Acad Sci U S A 109:21099-21103. CrossRef Medline

Sober SJ, Wohlgemuth MJ, Brainard MS (2008) Central contributions to acoustic variation in birdsong. J Neurosci 28:10370-10379. CrossRef Medline

Soha JA, Shimizu T, Doupe AJ (1996) Development of the catecholaminergic innervation of the song system of the male zebra finch. J Neurobiol 29:473-489. CrossRef Medline

Sohrabji F, Nordeen EJ, Nordeen KW (1990) Selective impairment of song learning following lesions of a forebrain nucleus in the juvenile zebra finch. Behav Neural Biol 53:51-63. CrossRef Medline

Surmeier DJ, Ding J, Day M, Wang Z, Shen W (2007) D1 and D2 dopaminereceptor modulation of striatal glutamatergic signaling in striatal medium spiny neurons. Trends Neurosci 30:228-235. CrossRef Medline

Tumer EC, Brainard MS (2007) Performance variability enables adaptive plasticity of 'crystallized' adult birdsong. Nature 450:1240-1244. CrossRef Medline

Turner RS, Desmurget M (2010) Basal ganglia contributions to motor control: a vigorous tutor. Curr Opin Neurobiol 20:704-716. CrossRef Medline

Vallentin D, Long MA (2015) Motor origin of precise synaptic inputs onto forebrain neurons driving a skilled behavior. J Neurosci 35:299-307. CrossRef Medline

Warren TL, Tumer EC, Charlesworth JD, Brainard MS (2011) Mechanisms and time course of vocal learning and consolidation in the adult songbird. J Neurophysiol 106:1806-1821. CrossRef Medline

Zhou QY, Palmiter RD (1995) Dopamine-deficient mice are severely hypoactive, adipsic, and aphagic. Cell 83:1197-1209. CrossRef Medline 